\title{
High-speed flow with discontinuous surface catalysis
}

\author{
By S. R. AMARATUNGA ${ }^{1}$, O. R. TUTTY ${ }^{2} \dagger$ \\ AND G. T. ROBERT $S^{2}$ \\ ${ }^{1}$ W. S. Atkins (Services) Ltd, Epsom KT18 5BW, UK \\ ${ }^{2}$ School of Engineering Sciences, University of Southampton, Southampton SO17 1BJ, UK
}

(Received 23 January 2000 and in revised form 6 June 2000)

In a reacting gas flow both gas-phase chemical activity and surface catalysis can increase the rate of heat transfer from the gas to a solid surface. In particular, when there is a discontinuous change in the catalytic properties of the surface, there can be a very large increase in the local heat transfer rate. In this study numerical simulations have been performed for the laminar high-speed flow of a high-temperature, non-equilibrium reacting gas mixture over a flat plate. The surface of the plate is partly catalytic, with the leading region non-catalytic, and a discontinuous change in the catalytic properties of the surface at the catalytic junction. The surface is assumed to be isothermal, and cold relative to the free stream. The gas is assumed to be a mixture of molecular and atomic forms of a diatomic gas in an inert gas forming a thermal bath, giving a three-species mixture with dissociation and recombination of the reactive species. The calculations are performed for a gas with atomic and molecular oxygen in an argon bath, but a full range of gas-phase chemical and surface catalytic effects is considered. Kinetic schemes with frozen gas-phase chemistry, and partial or full recombination of atomic oxygen in the boundary layer are investigated. The catalytic nature of the surface material is given by a catalytic recombination rate coefficient, which varies from zero (non-catalytic) to one (fully catalytic), and the effects on the flow and the surface heat transfer of materials which are non-, partially, or fully catalytic are considered. A self-similar thin-layer analytical model of the change in the gas composition downstream of the catalytic junction is developed. For physically realistic $\left(O\left(10^{-2}\right)\right)$ values of the catalytic recombination rate coefficient, the predictions from this model of the surface values of the atomic oxygen mass fraction and the catalytic surface heat transfer rate are excellent when the only change in the composition of the gas comes from the surface catalysis, and reasonable when there is partial recombination of the gas in the boundary layer due to the gas-phase chemistry. In contrast, when the surface is fully catalytic, the streamwise diffusion terms play a significant role, and the model is not valid. These results should apply to other situations with an attached boundary layer with recombination reactions. A comparison is made between the calculated and experimental measurements of the heat transfer rate at the catalytic junction. With a kinetic scheme which allows partial recombination in the boundary layer, good agreement is found between the experimental and predicted values for surface materials which are essentially non-catalytic. For a catalytic material (platinum), the experimental and numerical heat transfer rates are matched to estimate the value of the catalytic recombination rate coefficient. The values obtained show a considerable amount of scatter, but are consistent with those found in the literature. 


\section{Introduction}

In high-temperature, high-speed gas flow, dissociation and recombination of the constituent species of a gas mixture may occur. These finite-rate chemical reactions may have a significant effect on the flow. The reaction mechanism may alter considerably the chemical properties of the gas mixture which in turn affects the flow patterns, e.g. the location of shock waves, and surface quantities including heat transfer rates and pressure distribution. Further, even in a frozen non-equilibrium boundary layer, where the reactions occur at a slow rate relative to the speed of the flow so that the fluid travels through the flow field with no significant change in composition, it is possible for surface catalysis to occur, where the properties of the surface material enhance atom recombination. As a result of this surface influence, the chemical composition close to the surface may change significantly. This in turn may affect the thermodynamic state of the mixture near the surface. The exact mechanism of surface catalysis is complex, see e.g. Anderson (1989) or Dorrance (1962). The most important stages of the process have been summarized by Murray (1982) and Grumet, Anderson \& Lewis (1991) using the following steps:

(a) the transportation of reactants to the surface via diffusion;

(b) the adsorption of the reactants at the surface;

(c) chemical reaction between elements of the surface material and the reactants;

(d) the desorption of products at the surface;

(e) diffusion of products away from the surface.

The diffusion of species plays a key role in the process of surface catalysis, and, therefore, so must the transport properties of the constituents species of the gas. The extent to which catalytic effects influence the flow is also determined by the ability of the surface material to adsorb atoms and the rate at which surface recombination reactions occur.

The catalytic properties of a material for atom recombination is commonly measured by the catalytic recombination rate coefficient, or catalytic efficiency, $\gamma_{w}$, rather than the actual reaction rate. The catalytic recombination rate coefficient is defined as the fraction of the total number of atoms impinging on a unit surface area that recombine per unit time (see e.g. Goulard 1958 or Miller et al. 1995) and, therefore, has a range of 0 to 1 . The upper limit refers to a fully catalytic material where the surface reactions occur instantaneously, and the lower limit to a non-catalytic wall with no surface reactions. Any intermediate value refers to a partially catalytic material where the surface reactions are catalysed at a finite rate. The catalytic recombination rate coefficient is a temperature-dependent quantity, and a surface which is non-catalytic or partially catalytic at low temperature may be partially or fully catalytic at a higher temperature. Materials that are generally considered to be non-catalytic, e.g. ceramics and glass, have catalytic recombination rate coefficients of $O\left(10^{-3}-10^{-4}\right)$, while catalytic materials such as nickel, platinum, chromium, copper and gold may have catalytic recombination rate coefficients of $O\left(10^{-1}\right)$.

Atom recombination reactions seen in typical atmospheric gas mixtures are exothermic by nature and, therefore, the enhancement of such reactions by surface catalysis causes an increase in the surface heat transfer rate. Where there is a sharp change in the catalytic nature of the surface, the increase in the surface heat transfer rate may be particularly severe.

The effects of catalysis on surface heat transfer rates have been studied since at least the late 1950s by workers such as Lees (1956), Fay \& Riddell (1958), Goulard (1958), Chung \& Anderson (1961), and Inger (1963). Most of the early work concerned the 

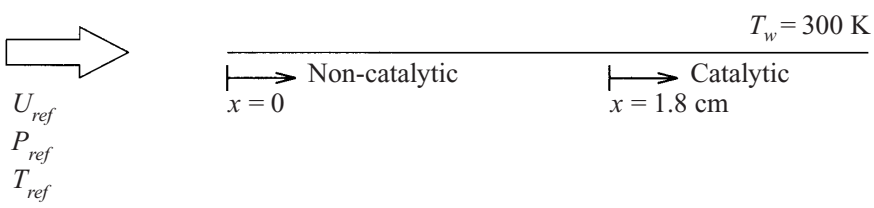

FIGURE 1. Experimental setup of the partly catalytic flat-plate test case.

stagnation-point heat transfer rate within a stream of dissociated air, with the main emphasis on reducing the diffusive surface heat flux by using non-catalytic material.

In the 1980s, a significant amount of the work in this area was aimed at the prediction and reduction of surface heating on the space shuttle through the use of non-catalytic materials (see e.g. Stewart, Rakich \& Lanfranco 1983 and Scott 1985 and references contained therein). Some more recent studies considered the effects of catalytic walls on a shock/boundary layer interaction (Grumet et al. 1991), catalytic effects on a model of Martian atmospheric entry (Chen \& Chandler 1993), and hypersonic flow past a sharp cone with finite catalytic walls (Miller et al. 1995).

This study investigates the effect of surface catalysis on the high-speed hightemperature flow of a gas mixture, the major component of which is an inert gas but with molecular and atomic forms of a diatomic gas. This gives a relatively simple three-species gas mixture, of the type used in experimental facilities, which allows dissociation and recombination of the diatomic species. The particular cases considered are of oxygen-argon mixtures over a flat plate with discontinuous surface catalysis. In addition to allowing a direct comparison with experimental results, the use of a specific gas mixture enables the use of standard methods to calculate parameters in the problem such as the various transport coefficients, rather than making perhaps unrealistic assumptions about the variation in these parameters with the composition of the gas mixture. However, a full range of chemical effects will be considered, covering non-catalytic, partially, and fully catalytic surfaces, and kinetic schemes ranging from frozen gas-phase chemistry so that the only change in the composition of the gas comes from surface effects, through schemes which allow partial recombination of oxygen in the boundary layer upstream of the catalytic region, to a scheme in which virtually all the atomic oxygen is removed before the flow reaches the catalytic surface. The last of course cannot involve surface catalysis since there is no atomic oxygen to recombine, but it does arise when using a kinetic scheme which is one of the most commonly used in studies of this kind.

There are two different sets of chemical reactions in the system considered here. The reactions in the flow are a set of homogeneous (gas-gas) reactions, while the surface catalysed reactions are heterogeneous (gas-solid). Throughout this study we will refer to the homogeneous reactions as gas-phase reactions while the heterogeneous reactions at the surface will be referred to as surface or catalytic reactions.

The test cases used here are those specified for the High Speed Flow Fields (HSFF) workshop held in Houston, Texas, in November 1995 (HSSF 1995). This is a set of 11 non-equilibrium reacting laminar supersonic flows of an oxygen-argon mixture over a partly catalytic flat plate, with a sharp leading edge. The free-stream Mach number is 2.4 , with a local deviation of no more than $\pm 2 \%$, and the free-stream Reynolds number is of order $3.5 \times 10^{3}$, based on a reference length of $1 \mathrm{~cm}$. At this Reynolds number the flow will be entirely laminar.

Figure 1 shows the experimental setup of the flat plate which consists of a noncatalytic region that extends for $1.8 \mathrm{~cm}$ from the leading edge followed by a partially 
catalytic surface region. The materials of interest are platinum, alumina and silicon monoxide. The first is catalytic while the last two are regarded as essentially noncatalytic at low temperatures. The free-stream temperature around $3000 \mathrm{~K}$ is relatively high compared to that of the wall which remains at approximately $300 \mathrm{~K}$ in the experiments due to the very short run times involved. Free-stream values of velocity, $U_{\infty}$, temperature, $T_{\infty}$, pressure, $p_{\infty}$, and species mole fraction, $X_{S_{\infty}}$, measured just ahead of the leading edge of the flat plate, were specified, as was a single heat transfer rate measurement, made at the leading edge of the catalytic material (hereafter referred to as the catalytic junction). Also given in the workshop specification was a reaction scheme.

The workshop proposal concerned code assessment in modelling gas-surface interactions of the steady state flow past the flat plate using a two-dimensional laminar, chemical-non-equilibrium CFD model. One of the basic requirements of the workshop was unusual, in that, instead of a straight comparison between experimental and numerical results, the objective was to match the numerical predictions of the surface heat transfer rate at the catalytic junction to the given experimental values by determining appropriate surface catalytic recombination rate coefficients.

This paper considers the problem of a flat plate with discontinuous catalytic behaviour, although the major results should apply in more general situations. The behaviour of the flow in the region of the discontinuity on the surface is studied using a combination of numerical results and analytical models. In addition, predictions are made of the recombination rate coefficient. Below, we give the governing equations, including the reaction scheme and the surface catalytic model, the test cases, a brief description of the key features of the numerical scheme, a detailed study of one case where the surface effects are expected to be particularly significant, predictions of the catalytic recombination rate coefficients, and, finally, a discussion of the results.

The code used for this study works with the governing equations cast in dimensionless form. However, given the complexity of the models used, it is much easier to present the formulation of the problem in dimensional form, which will be used throughout. For the most part SI units have been used. This includes moles as $\mathrm{kg}$-moles rather than gm-moles as is commonly used in chemistry.

\section{Governing equations}

The governing equations for a compressible, reactive flow can be written in conservative form in Cartesian coordinates as

$$
\frac{\partial \boldsymbol{U}}{\partial t}+\frac{\partial \boldsymbol{F}}{\partial x}+\frac{\partial \boldsymbol{G}}{\partial y}=\frac{\partial \boldsymbol{F}_{v}}{\partial x}+\frac{\partial \boldsymbol{G}_{v}}{\partial y}+\dot{\boldsymbol{\omega}},
$$

where

$$
\begin{gathered}
\boldsymbol{U}=\left(\begin{array}{c}
\rho_{s} \\
\rho u \\
\rho v \\
\rho E
\end{array}\right), \\
\boldsymbol{F}=\left(\begin{array}{c}
\rho_{s} u \\
\rho u^{2}+p \\
\rho u v \\
u(\rho E+p)
\end{array}\right), \boldsymbol{G}=\left(\begin{array}{c}
\rho_{s} v \\
\rho u v \\
\rho v^{2}+p \\
v(\rho E+p)
\end{array}\right),
\end{gathered}
$$




$$
\begin{gathered}
\boldsymbol{F}_{v}=\left(\begin{array}{c}
-\rho_{s} u_{D s} \\
\tau_{x x} \\
\tau_{x y} \\
u \tau_{x x}+v \tau_{x y}-q_{x}
\end{array}\right), \\
\boldsymbol{G}_{v}=\left(\begin{array}{c}
-\rho_{s} v_{D s} \\
\tau_{x y} \\
\tau_{y y} \\
u \tau_{x y}+v \tau_{y y}-q_{y}
\end{array}\right), \\
\dot{\boldsymbol{\omega}}=\left(\dot{\omega}_{s}, 0,0,0\right)^{T} .
\end{gathered}
$$

Here the subscript $s$ denotes a species in the mixture, $\rho_{s}$ are the species densities, $\rho=\sum_{s=1}^{N_{s}} \rho_{s}$ is the total density of the mixture, $\boldsymbol{u}=(u, v)$ is the bulk velocity of the mixture and $\boldsymbol{u}_{D s}=\left(u_{D s}, v_{D s}\right)$ the species diffusion velocities in Cartesian coordinates $(x, y), p$ is the pressure, $E$ the total specific energy of the gas mixture, $\boldsymbol{q}=\left(q_{x}, q_{y}\right)$ is the heat flux, and the $\dot{\omega}_{s}$ are the species source terms from the chemical reactions. There are $N_{s}$ species and therefore $N_{s}$ species continuity equations.

As usual, the shear stress tensor is given by

$$
\tau_{x x}=2 \mu \frac{\partial u}{\partial x}-\frac{2}{3} \nabla \cdot \boldsymbol{u}, \quad \tau_{x y}=\mu\left(\frac{\partial u}{\partial y}+\frac{\partial v}{\partial x}\right), \quad \tau_{y y}=2 \mu \frac{\partial v}{\partial y}-\frac{2}{3} \nabla \cdot \boldsymbol{u},
$$

where $\mu$ is the viscosity of the mixture. The heat flux term for a chemically reacting flow is given by

$$
\boldsymbol{q}=-\lambda \nabla T+\sum_{s=1}^{N_{s}} h_{s} \rho_{s} \boldsymbol{u}_{D s},
$$

where $\lambda$ is the thermal conductivity of the mixture, $T$ is the temperature, and the $h_{s}$ are the species enthalpies.

Equations (1)-(8) give the full Navier-Stokes equations for a compressible, viscous, reacting gas flow. Commonly in numerical studies of this kind with a high-speed flow producing an attached boundary layer, the thin-layer form of the NavierStokes equations is used, i.e. $\boldsymbol{F}_{v}$ is dropped as are terms in $\boldsymbol{G}_{v}$ which give rise to cross-derivatives. Usually this makes little difference to the results, and is more computationally efficient. However, in this study, as will be seen below, in certain cases the streamwise diffusion must be included, although the cross-derivative terms could have been safely omitted.

The first component of the viscous flux vectors, $\boldsymbol{F}_{v}$ and $\boldsymbol{G}_{v}$, represents the mass diffusion flux of species $s$ which is assumed to obey Fick's law, giving

$$
\rho_{s} \boldsymbol{u}_{D s}=-\frac{\mu L e}{P r} \nabla Y_{s}
$$

where $Y_{s}=\rho_{s} / \rho$ is the species mass fraction. Note that (9) automatically satisfies the requirement $\sum_{s=1}^{N_{s}} \rho_{s} \boldsymbol{u}_{D s} \equiv 0$. The Lewis number, Le, is assumed constant for any particular run, but a sensitivity analysis of the effects of a change in Le has been performed, and the results are reported below. The Prandtl number is given by

$$
\operatorname{Pr}=\frac{\mu c_{p}}{\lambda}
$$

where $c_{p}$ is the mixture specific heat at constant pressure. In this study the Prandtl number is not fixed but calculated from (10).

The constituent gases that make up the mixture are assumed to behave ideally, and 
(a)

\begin{tabular}{cccc}
\cline { 2 - 4 } Coefficient & $\mathrm{O}$ & $\mathrm{O}_{2}$ & $\mathrm{Ar}$ \\
$a_{1}$ & 2.6428 & 3.267 & 2.5000 \\
$a_{2}$ & $-1.7596 \times 10^{-4}$ & $1.1324 \times 10^{-3}$ & 0.0 \\
$a_{3}$ & $6.075 \times 10^{-8}$ & $-2.7934 \times 10^{-7}$ & 0.0 \\
$a_{4}$ & $-5.2372 \times 10^{-12}$ & $2.5253 \times 10^{-11}$ & 0.0 \\
$a_{5}$ & $-5.0993 \times 10^{-18}$ & $2.0093 \times 10^{-17}$ & 0.0 \\
$a_{6}$ & $2.9215 \times 10^{4}$ & $-1.0243 \times 10^{3}$ & $-7.45375 \times 10^{-2}$ \\
& & $(b)$ & \\
Coefficient & $\mathrm{O}$ & $\mathrm{O} 2$ & $\mathrm{Ar}$ \\
$a_{1}$ & 2.3584 & 2.2893 & 0.0 \\
$a_{2}$ & $8.8833 \times 10^{-5}$ & $6.7967 \times 10^{-4}$ & 0.0 \\
$a_{3}$ & $-9.4390 \times 10^{-9}$ & $-2.3506 \times 10^{-8}$ & 0.0 \\
$a_{4}$ & $4.5843 \times 10^{-13}$ & $1.1871 \times 10^{-13}$ & 0.0 \\
$a_{5}$ & $1.7023 \times 10^{-20}$ & $-5.3044 \times 10^{-20}$ & $-7.45375 \times 10^{2}$ \\
$a_{6}$ & $2.9356 \times 10^{4}$ & $2.8033 \times 10^{3}$ &
\end{tabular}

TABLE 1. Thermodynamic curve fit coefficients (in SI units) for (a) $300 K<T<5000 K$,

(b) $5000 \mathrm{~K}<T<25000 \mathrm{~K}$

hence the equation of state for the gas mixture is given by

$$
p=\sum_{s=1}^{N_{s}} \rho_{s} R_{s} T \equiv \rho R_{0} T \sum_{s=1}^{N_{s}} Y_{i} / M_{s},
$$

where $R=\sum_{s=1}^{N_{s}} R_{s} Y_{s}$ is the gas constant of the mixture, $R_{s}=R_{0} / M_{s}$ is the species gas constant, $R_{0}$ is the universal gas constant, and the $M_{s}$ are the molar masses of the species.

The temperature is obtained by solving (iteratively) the energy equation

$$
E=\sum_{s=1}^{N_{s}} Y_{s} h_{s}-R T+\frac{1}{2}\left(u^{2}+v^{2}\right),
$$

where the species enthalpies, $h_{s}$, are expressed as a polynomial function of temperature

$$
h_{s}=R_{s}\left(a_{1 s} T+\frac{a_{2 s}}{2} T^{2}+\frac{a_{3 s}}{3} T^{3}+\frac{a_{4 s}}{4} T^{4}+\frac{a_{5 s}}{5} T^{5}+a_{6 s}\right) .
$$

The coefficients in (13) for the $\mathrm{O}$ and $\mathrm{O}_{2}$ enthalpy curve fits are obtained from JANAF thermodynamic data tables (Stull \& Prophet 1971) for a temperature range of $300 \mathrm{~K}$ to $5000 \mathrm{~K}$ (table $1 a$ ) and from Balakrishnan (1986) for a temperature range of $5000 \mathrm{~K}$ to $25000 \mathrm{~K}$ (table $1 b$ ). Thermodynamic data are obtained for Ar from the Chemkin data base (Kee, Rupley \& Miller 1987). A similar polynomial expression is obtained for species specific heat at constant pressure $\left(c_{p s}\right)$ by differentiating the species enthalpy relation with respect to temperature, i.e. $c_{p s}=\partial h_{s} /\left.\partial T\right|_{p}$.

\section{Transport properties}

Species viscosities are expressed in SI units as a function of the Lennard-Jones parameters (table 2), $\sigma_{s}$ and $\epsilon_{s} / k$ as suggested by Reid, Prausnitz \& Sherwood (1977), 


$\begin{array}{ccc}\text { Species } & \sigma_{i}(\AA) & \epsilon_{i} / k(\mathrm{~K}) \\ \mathrm{O} & 3.050 & 106.7 \\ \mathrm{O}_{2} & 3.467 & 106.7 \\ \mathrm{Ar} & 3.542 & 93.3\end{array}$

TABLE 2. Lennard-Jones parameters

i.e.

$$
\mu_{s}=2.6693 \times 10^{-6} \frac{\sqrt{M_{s} T}}{\sigma_{s}^{2} \Omega_{\mu_{s}}},
$$

where $\Omega_{\mu s}$ is the species viscosity collision integral, $\sigma_{s}$ is the molecular diameter measured in $\AA$, and $\epsilon_{s} / k$ is a reference temperature calculated from $\epsilon_{s}$, the depth of the potential well in the Lennard-Jones (12-6) potential, and Boltzmann's constant $k$. Species collision integrals are expressed as functions of the reduced temperature

$$
T^{*}=\frac{T}{\epsilon_{s} / k}
$$

given by Reid et al. (1977) as

$$
\Omega_{\mu_{i}}=A T^{*-B}+C \mathrm{e}^{-D T^{*}}+E \mathrm{e}^{-F T^{*}},
$$

where the constants are $A=1.16145, B=0.14874, C=0.52487, D=0.77320$, $E=2.16178$ and $F=2.43787$. This formulation applies to a temperature range of $0.3<T^{*}<100$.

The thermal conductivity for each species is obtained from Euken's relation (Vincenti \& Kruger 1965) to correct the energy transfer between the internal structure and the translational motion

$$
\lambda_{s}=\mu_{s}\left(c_{v s}+\frac{9}{4} \frac{R_{0}}{M_{s}}\right) .
$$

The mixture transport properties are calculated using Wilke's (1950) mixing rule

$$
\begin{aligned}
& \mu=\sum_{s=1}^{N_{s}} \mu_{s} \frac{Y_{s}}{M_{s}} \frac{1}{\sum_{k=1}^{N_{s}}\left(Y_{k} / M_{k}\right) A_{s, k}}, \\
& \lambda=\sum_{s=1}^{N_{s}} \lambda_{s} \frac{Y_{s}}{M_{s}} \frac{1}{\sum_{k=1}^{N_{s}}\left(Y_{k} / M_{k}\right) A_{s, k}},
\end{aligned}
$$

where

$$
A_{s, k}=\frac{1}{2 \sqrt{2}} \frac{\left[1+\left(\mu_{s} / \mu_{k}\right)^{1 / 2}\left(M_{k} / M_{s}\right)^{1 / 4}\right]^{2}}{\left[1+M_{s} / M_{k}\right]^{1 / 2}} .
$$

\section{Surface conditions}

The conditions on the flat plate are the standard viscous conditions with zero velocity and an isothermal wall with $T_{w}=300 \mathrm{~K}$, supplemented by the catalytic model on the downstream $(x>1.8 \mathrm{~cm})$ portion of the plate. The process of catalysis describes the transport of species at the surface of the plate due to the properties 
of the catalytic material. This reduces to a specific boundary condition for the mass diffusion terms in the viscous flux vector, $\boldsymbol{G}_{v}$, at the catalytic wall, given by

$$
\left.\begin{array}{l}
\mu \frac{\operatorname{Le}}{\operatorname{Pr}}\left(\frac{\partial Y_{O}}{\partial y}\right)_{w}=\gamma_{w}\left(\frac{R_{0} T_{w}}{2 \pi M_{O}}\right)^{1 / 2} \rho_{w} Y_{O}, \\
\mu \frac{L e}{\operatorname{Pr}}\left(\frac{\partial Y_{O_{2}}}{\partial y}\right)_{w}=-\gamma_{w}\left(\frac{R_{0} T_{w}}{2 \pi M_{O}}\right)^{1 / 2} \rho_{w} Y_{O}, \\
\mu \frac{\operatorname{Le}}{\operatorname{Pr}}\left(\frac{\partial Y_{A r}}{\partial y}\right)_{w}=0,
\end{array}\right\}
$$

where $\gamma_{w}$ is the catalytic rate coefficient. It varies from zero for a non-catalytic surface through to one for a fully catalytic surface. Note that this is not the only definition that could be used for a fully catalytic wall (see e.g. Anderson 1989), but it is the appropriate one here.

\section{Reaction mechanism}

A three-species $\left(\mathrm{O}_{2}, \mathrm{O}, \mathrm{Ar}\right)$, three reaction mechanism will be used in the gas:

$$
\left.\begin{array}{rl}
\mathrm{O}_{2}+\mathrm{O}_{2} & \rightleftharpoons \mathrm{O}+\mathrm{O}+\mathrm{O}_{2}, \\
\mathrm{O}_{2}+\mathrm{O} & \rightleftharpoons \mathrm{O}+\mathrm{O}+\mathrm{O}, \\
\mathrm{O}_{2}+\mathrm{Ar} & \rightleftharpoons \mathrm{O}+\mathrm{O}+\text { Ar. }
\end{array}\right\}
$$

Given expressions for the forward and backward reaction rates for each reaction, $K_{f r}$ and $K_{b r}$ respectively where $r$ is the reaction number, the sum of the rates of production of a species over each non-equilibrium reaction gives the net rate of production of that species, so that

$$
\dot{\omega}_{s} \equiv \frac{\mathrm{d} \rho_{s}}{\mathrm{~d} t}=M_{s} \sum_{r=1}^{N_{r}}\left(v_{s r}^{\prime}-v_{s r}^{\prime \prime}\right)\left[K_{b r} \prod_{k=1}^{N_{s}}\left(\frac{\rho_{k}}{M_{k}}\right)^{v_{k r}^{\prime \prime}}-K_{f r} \prod_{k=1}^{N_{s}}\left(\frac{\rho_{k}}{M_{k}}\right)^{v_{k r}^{\prime}}\right],
$$

where $v_{s r}^{\prime}$ and $v_{s r}^{\prime \prime}$ are the stoichiometric coefficients of the reactants and products respectively of species $s$ in reaction $r$. The sum of the species source terms, $\dot{\omega}_{s}$, is identically zero.

In the literature a large number of different sets of forward and reverse reaction rate constants, $K_{b f}$ and $K_{b r}$, can be found for oxygen dissociation/recombination reaction schemes, some of which will be considered here. In the problem definition for the Houston workshop (HSFF 1995), forward and backward reaction rate constants were specified in Arrhenius form as

$$
\begin{gathered}
K_{f}=A T^{a} \mathrm{e}^{-E_{a} / R T}, \\
K_{b}=B T^{b} .
\end{gathered}
$$

Note that in the workshop specification the exponential term, $\exp \left(-E_{a} / R T\right)$, was included in both the forward and backward rate constants. This is clearly an error as it implies the same activation energy for the recombination as for the dissociation of oxygen, whereas a zero activation energy is expected for the recombination. Further, for the problem considered here, including this term in (24) produces in effect a chemically frozen boundary layer, i.e. no significant reactions in the gas phase. Hence the exponential term has been dropped in the expression for the backward rate constant. The constants $A, a, E_{a} / R, B$ and $b$ are given in table $3(a)$. 


\begin{tabular}{|c|c|c|c|c|c|c|}
\hline & Reaction & $A$ & $a$ & $E_{a} / R$ & $B$ & $b$ \\
\hline \multirow[t]{3}{*}{ (a) } & $\mathrm{O}_{2}+\mathrm{O}_{2} \rightleftharpoons \mathrm{O}+\mathrm{O}+\mathrm{O}_{2}$ & $23.0 \times 10^{15}$ & -1 & 59400 & $19.0 \times 10^{9}$ & $-\frac{1}{2}$ \\
\hline & $\mathrm{O}_{2}+\mathrm{O} \rightleftharpoons \mathrm{O}+\mathrm{O}+\mathrm{O}$ & $85.0 \times 10^{15}$ & -1 & 59400 & $71.0 \times 10^{9}$ & $-\frac{1}{2}$ \\
\hline & $\mathrm{O}_{2}+\mathrm{Ar} \rightleftharpoons \mathrm{O}+\mathrm{O}+\mathrm{Ar}$ & $3.0 \times 10^{15}$ & -1 & 59400 & $2.5 \times 10^{9}$ & $-\frac{1}{2}$ \\
\hline \multirow[t]{3}{*}{ (b) } & $\mathrm{O}_{2}+\mathrm{O}_{2} \rightleftharpoons \mathrm{O}+\mathrm{O}+\mathrm{O}_{2}$ & $27.5 \times 10^{15}$ & -1 & 59500 & & \\
\hline & $\mathrm{O}_{2}+\mathrm{O} \rightleftharpoons \mathrm{O}+\mathrm{O}+\mathrm{O}$ & $82.5 \times 10^{15}$ & -1 & 59500 & & \\
\hline & $\mathrm{O}_{2}+\mathrm{Ar} \rightleftharpoons \mathrm{O}+\mathrm{O}+\mathrm{Ar}$ & $3.0 \times 10^{15}$ & -1 & 59500 & & \\
\hline \multirow[t]{3}{*}{ (c) } & $\mathrm{O}_{2}+\mathrm{O}_{2} \rightleftharpoons \mathrm{O}+\mathrm{O}+\mathrm{O}_{2}$ & $32.0 \times 10^{15}$ & -1 & 59500 & & \\
\hline & $\mathrm{O}_{2}+\mathrm{O} \rightleftharpoons \mathrm{O}+\mathrm{O}+\mathrm{O}$ & $20.0 \times 10^{15}$ & -1 & 59500 & & \\
\hline & $\mathrm{O}_{2}+\mathrm{Ar} \rightleftharpoons \mathrm{O}+\mathrm{O}+\mathrm{Ar}$ & $3.0 \times 10^{15}$ & -1 & 59500 & & \\
\hline
\end{tabular}

TABle 3. Reaction rate constants from $(a)$ the HSFF workshop, (b) Park, and $(c)$ Kang \& Dunn. $A$ has units $\mathrm{m}^{3} \mathrm{~mol}^{-1} \mathrm{~s}^{-1} \mathrm{~K}$ and $B \mathrm{~m}^{6} \mathrm{~mol}^{-2} \mathrm{~s}^{-1} \mathrm{~K}^{1 / 2}$.

Some of the most commonly used reaction schemes for high-speed, high-temperature flow are those given by Park $(1984,1985)$. These schemes give the forward rate constants in standard Arrhenius form, and the equilibrium constant, $K_{e}$ in the form

$$
K_{e}(T)=\exp \left(A_{1}+A_{2} Z+A_{3} Z^{2}+A_{4} Z^{3}+A_{5} Z^{4}\right),
$$

where $Z=10000 / T$. The backward rate is then calculated from

$$
K_{b}=\frac{K_{f}}{K_{e}} .
$$

For oxygen recombination reactions, as in (22), Park (1985) gives $A_{1}=8.243, A_{2}=$ $-4.127, A_{3}=-0.616, A_{4}=0.093$ and $A_{5}=-0.005$, where $K_{e}$ has units $\mathrm{m}^{-3} \mathrm{~mol}$. Park (1985) gives reaction rate constants for the dissociation of oxygen with molecular and atomic oxygen as the collision partner (the first two forward reactions of (22)), which are similar to the rates given in table $3(a)$, but not for the third reaction as Park's reaction schemes do not involve argon. For this reaction we use essentially the same forward rate constant as in table $3(a)$. With this assumption, the forward rate constants for Park's scheme are listed in table $3(b)$.

Park (1984) gives a second set of values for the first two of the forward reactions, quoted from Kang \& Dunn (1972). With the same forward rate constant for the third reaction as before, these rate constants are given in table $3(c)$.

The rate constants given above appear to be essentially for high-temperature flows, particularly for the backward reactions (this will be discussed further below). Chung (1965) gives rate constants for oxygen recombination reactions in the form

$$
K_{b}=A T^{-3 / 2},
$$

where $A=0.4 \times 10^{14}$ or $1.125 \times 10^{14} \mathrm{~m}^{6} \mathrm{~mol}^{-2} \mathrm{~s}^{-1} \mathrm{~K}^{3 / 2}$ where, respectively, molecular or atomic oxygen is the collision partner. These could be used directly with the forward rate constants given above to produce further reaction schemes. However, these schemes would not be consistent in that they would have different equilibrium constants for at least the first two reactions in (21). Taking the forward reaction rate constants in table $3(a)$ as a guide and using the same equilibrium constant for each reaction, then with the free-stream species mass fractions used in this study (see table 5 below), the second reaction with atomic oxygen as the collision partner will be the 
most important recombination reaction. Hence the expression given by Chung (1965) for this reaction was used to define an an equilibrium constant in the form

$$
K_{e}=K_{f_{o}} / 1.125 \times 10^{14} T^{-3 / 2},
$$

where $K_{f_{o}}$ is a forward rate constant with atomic oxygen as the collision partner. Given a set of forward rate constants, (28) can now be used to calculate the backward rate constants. Alternatively, the backward rate constants given by Chung for atomic and molecular oxygen could be combined with a backward rate constant for argon and then used with a equilibrium constant, e.g (25), to produce a set a forward rate constants. Both of these approaches have been tried.

We now have a number of sets of reaction rates which we could use. However, before continuing it is worth examining in general terms the likely effects of these various sets, particularly as regards recombination of oxygen in the boundary layer, as this will directly affect the heat transfer to the surface in the catalytic region. Park's scheme (table $3(b)$ and (25)) gives a forward rate constant of $O\left(10^{-72}\right.$ ) and a reverse rate constant of $O\left(10^{1466}\right)$ at $300 \mathrm{~K}$, i.e. in effect no dissociation and an infinite backward rate giving instantaneous recombination as soon as the gas enters the boundary layer. In this case, there cannot be any surface catalysis as there will be no atomic oxygen in the flow by the time the flow reaches the catalytic region. Further, with reverse reaction rates of this order, there would be no surface catalysis at any realistic flow speed, which is unlikely physically. In contrast, Chung's expression (27) produces reverse rate constants of $O\left(10^{10}\right)$ at $300 \mathrm{~K}$, which would allow a degree of recombination of oxygen in the boundary layer.

Park (1985) states that the coefficients for the equilibrium constant were obtained from a fit to spectroscopic data at 1000, 2000, 4000, 8000 and $16000 \mathrm{~K}$. Examination of Park's fit shows that while $\ln K_{e}$ is close to linear in $Z$ for $1000 \leqslant T \leqslant 16000$, it rapidly deviates from this for $T \leqslant 1000$. This is not surprising as polynomial fits of maximum possible order for the data available are frequently poorly behaved when used for extrapolation. Here, although the coefficient is small, the last term in (25) dominates below $1000 \mathrm{~K}$. A simpler alternative form of $K_{e}$ consistent with Park's for $1000 \leqslant T \leqslant 16000$ was produced by making a linear curve fit to the values produced by (25) at the temperature listed above. The straight line derived gives a very close fit through the data points, with a (negative) correlation coefficient greater than 0.999 , and matches the values from (25) closely throughout $1000 \leqslant T \leqslant 16000$, but does not produce unrealistically small values of $K_{e}$ at low temperatures. This fit is

$$
K_{e}=\exp (10.111-61110 / T) .
$$

Note that, as with Park's original method, this gives a small activation energy.

Values of the reaction rate constants at 300 and $3500 \mathrm{~K}$, which is basically the range of interest here, with atomic oxygen as the collision partner are given in table 4 . The notation for the reaction schemes is $\mathrm{H}$ for the HSFF workshop values (table $3 a$ ), $\mathrm{P}$ for Parks scheme (table $3 b$ or (25)), PM when using the modified form for $K_{e},(29)$, KD for Kang \& Dunn's values (table $3 c$ ), C1 for Chung's reverse reaction scheme using (28), and $\mathrm{C} 2$ for Chung's scheme using the rate constants given by Chung for molecular and atomic oxygen as the collision partner and backward rate from the Houston scheme for argon. These are combined so that e.g. KD:PM refers to Kang $\&$ Dunn for the forward rates and $K_{e}$ from (29) for the backward reactions.

Also given in table 4 are values of $\ln K_{e}$ where $K_{e}$ is obtained from (25), (28) and (29), or calculated using (26). The equilibrium constant is not as such a property of the kinetics of a reaction scheme but a basic thermodynamic quantity, and in theory can 


\begin{tabular}{cccccccc}
\hline & \multicolumn{3}{c}{$300 \mathrm{~K}$} & & \multicolumn{3}{c}{$3500 \mathrm{~K}$} \\
\cline { 3 - 4 } \cline { 5 - 6 } $\begin{array}{c}\text { Reaction } \\
\text { scheme }\end{array}$ & $K_{f}$ & $K_{b}$ & $\ln K_{e}$ & & $K_{f}$ & $K_{b}$ & $\ln K_{e}$ \\
H:H & $2.9 \times 10^{-72}$ & $4.1 \times 10^{9}$ & -186.86 & & $1.0 \times 10^{6}$ & $1.2 \times 10^{9}$ & -7.06 \\
P:P & $2.0 \times 10^{-72}$ & $4.4 \times 10^{1466}$ & -3542.07 & & $9.8 \times 10^{5}$ & $8.3 \times 10^{8}$ & -6.74 \\
P:PM & $2.0 \times 10^{-72}$ & $2.4 \times 10^{12}$ & -193.59 & & $9.8 \times 10^{5}$ & $1.5 \times 10^{9}$ & -7.35 \\
KD:PM & $4.9 \times 10^{-73}$ & $5.8 \times 10^{11}$ & -193.59 & & $2.4 \times 10^{5}$ & $3.7 \times 10^{8}$ & -7.35 \\
P:C1 & $2.0 \times 10^{-72}$ & $2.2 \times 10^{10}$ & -188.88 & & $9.8 \times 10^{5}$ & $5.4 \times 10^{8}$ & -6.32 \\
KD:C1 & $4.9 \times 10^{-73}$ & $2.2 \times 10^{10}$ & -190.30 & & $2.4 \times 10^{5}$ & $5.4 \times 10^{8}$ & -7.74 \\
PM:C2 & $1.8 \times 10^{-74}$ & $2.2 \times 10^{10}$ & -193.59 & & $3.5 \times 10^{5}$ & $5.4 \times 10^{8}$ & -7.35 \\
JANAF & & & -188.95 & & & & -7.09
\end{tabular}

TABLE 4. Reaction rate and equilibrium constants for the different schemes with atomic oxygen as the collision partner.

be calculated from knowledge of the basic state of the system under consideration. At equilibrium the forward and backward rates match, and consequently (26) must hold. A standard assumption, used here, is that (26) can be used away from equilibrium to determine forward or backward rate constants given $K_{e}$ and rate constants in one direction. It follows that any kinetic scheme which does not use or produce a reasonable value of $K_{e}$ is almost certainly wrong. Calculated values of $\log K_{p}$, where $K_{p}$ is the equilibrium constant in terms of partial pressure of the components of the gas mixture (here $K_{p}=R_{0} T K_{e}$ ), are tabulated in the JANAF tables (Stull \& Prophet 1971). Note that the formula used to calculate the theoretical values for $\log K_{p}$ in the JANAF tables involves $\log K_{p}$ rather than $K_{p}$, and by convention, equilibrium constants are tabulated in logarithmic form.

From table 4, it appears that apart from the reverse reaction from Park's original scheme at $300 \mathrm{~K}$, which is clearly wrong, all the reaction schemes produce reasonably consistent values. There is still however considerable variation in the reaction rate constants, reflecting the uncertainty in the values for reactions of this kind. At $300 \mathrm{~K}$, the values of $\ln K_{e}$ are, apart from Park's scheme, within $2.5 \%$ of the JANAF values. This is consistent with the difference between the calculated and observed values of $\log K_{p}$ given in the sample calculations presented in the JANAF tables. Note in particular that the modified form of Park's equilibrium constant, (29), produces a reasonable value although it involves extrapolation to a low temperature. There appears to be proportionally more spread in the values of $\ln K_{e}$ at $3500 \mathrm{~K}$, although this reflects in part the fact that this is not far from the point where $\ln K_{e}$, or more precisely, $\log K_{p}$, goes through zero.

In summary, it appears that all the sets of rate constants except Park's original scheme could be used in an attempt to calculate the flows under consideration. Park's original scheme is still of some theoretical interest as it will produce the case in which there is complete recombination in the boundary layer.

\section{Test cases}

A set of 11 test cases of non-equilibrium laminar flow over a partly catalytic flat plate will be considered. The materials of interest are platinum $(\mathrm{Pt})$, alumina $\left(\mathrm{Al}_{2} \mathrm{O}_{3}\right)$ and silicon monoxide $(\mathrm{SiO})$. The first of these is catalytic, but the others are regarded as being essentially non-catalytic at low temperatures. The free-stream conditions and 


\begin{tabular}{ccccccccc}
\hline & & $U_{\infty}$ & $T_{\infty}$ & $p_{\infty}$ & & & & $q_{w}$ \\
Case & Material & $\left(\mathrm{ms}^{-1}\right)$ & $\mathrm{K}$ & $\mathrm{N} \mathrm{m}^{-2}$ & $Y_{O}$ & $Y_{O_{2}}$ & $Y_{A r}$ & $10^{6} \mathrm{~W} \mathrm{~m}^{-2}$ \\
1 & $\mathrm{Pt}$ & 2760 & 3180 & 10531 & 0.142 & 0.029 & 0.829 & 1.675 \\
2 & $\mathrm{Pt}$ & 2740 & 3180 & 11997 & 0.142 & 0.029 & 0.829 & 2.102 \\
3 & $\mathrm{Pt}$ & 2830 & 3300 & 11597 & 0.147 & 0.020 & 0.833 & 1.759 \\
4 & $\mathrm{Pt}$ & 2590 & 3020 & 10397 & 0.119 & 0.048 & 0.833 & 1.553 \\
5 & $\mathrm{Al}_{2} \mathrm{O}_{3}$ & 2800 & 3240 & 11197 & 0.147 & 0.020 & 0.833 & 1.382 \\
6 & $\mathrm{SiO}$ & 2790 & 3240 & 11864 & 0.147 & 0.020 & 0.833 & 1.436 \\
7 & $\mathrm{SiO}$ & 2820 & 3280 & 11331 & 0.147 & 0.020 & 0.833 & 1.491 \\
8 & $\mathrm{SiO}$ & 2820 & 3290 & 12797 & 0.147 & 0.020 & 0.833 & 1.553 \\
9 & $\mathrm{SiO}$ & 2800 & 3240 & 11197 & 0.147 & 0.020 & 0.833 & 1.465 \\
10 & $\mathrm{SiO}$ & 2830 & 3310 & 12264 & 0.147 & 0.020 & 0.833 & 1.541 \\
11 & $\mathrm{SiO}$ & 2760 & 3180 & 10931 & 0.147 & 0.020 & 0.833 & 1.319
\end{tabular}

TABLE 5. Free-stream conditions and the surface heat transfer rate at the catalytic junction $(x / L=1.8)$ for the eleven test cases.

species fractions are specified for each test case in table 5 , as is the measured value of the heat transfer rate at the catalytic junction. These values have been converted from the form given in the workshop - mole fraction for the species, pressures in $\mathrm{mm}$ $\mathrm{Hg}$, and $q_{w}$ at the catalytic junction in cal cm $\mathrm{cm}^{-2} \mathrm{~s}^{-1}$ to the form used in this study, mass fraction, $\mathrm{N} \mathrm{m}^{-2}$ and $\mathrm{W} \mathrm{m}^{-2}$.

\section{Numerical scheme}

The numerical scheme adopts a convection-diffusion-reaction operator splitting sequence following Batten et al. (1996) to account for inviscid, viscous and reactive contributions to the flow, giving, in turn,

$$
\begin{gathered}
\frac{\partial \boldsymbol{U}}{\partial t}+\frac{\partial \boldsymbol{F}}{\partial x}+\frac{\partial \boldsymbol{G}}{\partial y}=0, \\
\frac{\partial \boldsymbol{U}}{\partial t}=\frac{\partial \boldsymbol{F}_{v}}{\partial x}+\frac{\partial \boldsymbol{G}_{v}}{\partial y}, \\
\frac{\partial \boldsymbol{U}}{\partial t}=\dot{\boldsymbol{\omega}} .
\end{gathered}
$$

This allows independent numerical schemes to be used on each of the above PDEs for the individual contributory sources, which can be combined to advance the entire solution in time. Here a brief description of the main features of the method will be given, along with some details where they played a significant role in this study. Further details of the method can be found in Amaratunga (1998). The code used is a full unsteady solver, and the results presented below were generated by time marching to a steady state. The code was developed specifically for distributed memory parallel processor arrays. In fact this was a major reason for adopting the operator splitting approach as it is well matched to this architecture, as simple domain decomposition can be used where the domain is divided in the streamwise direction into blocks with an equal number of grid points. There is mainly local coupling between grid points, and as a result relatively low communication overheads, consisting primarily of end swaps between the adjacent processors. Hence it is straightforward to obtain good parallel efficiency. 
The inviscid operator (30) is itself split into one-dimensional equations in the coordinate directions which are then solved using a Godunov-type method (Godunov 1959). The method employs a three-wave HLLC approximate Riemann solver (Toro, Spruce \& Spears 1994; Batten et al. 1996). In addition, a MUSCL reconstruction (Van Leer 1979) of the data was used to increase the spatial resolution of the scheme. In an early version of this work, an attempt was made to use the simpler two-wave HLLE (Harten, Lax \& Van Leer 1983; Einfeldt 1988) Riemann solver, but this method was discarded due to inaccuracies in the results near the solid wall. In particular, for a flat-plate boundary layer with an adiabatic wall condition, when using the HLLE scheme the calculated recovery temperature on the wall was much larger than expected.

The viscous contribution to the flow was calculated in different ways depending on the problem. The common element was a one-step linearized implicit method for the transverse diffusion terms. Taking $\boldsymbol{G}_{t}$ as the vector of elements of $\boldsymbol{G}_{v}$ which contain derivatives in $y$ only, then the vector of changes to the variables, $\delta \boldsymbol{U}$, is given by the solution of

$$
\left[\boldsymbol{I}-\Delta t\left(\frac{\partial \hat{\boldsymbol{G}}_{t}}{\partial \boldsymbol{U}}\right)^{n}\right] \delta \boldsymbol{U}=\Delta t \hat{\boldsymbol{G}}_{t}^{n},
$$

where $n$ is the time step,

$$
\hat{\boldsymbol{G}}_{t}^{n}=\frac{\boldsymbol{G}_{t j+1 / 2}^{n}-\boldsymbol{G}_{t j-1 / 2}^{n}}{\Delta y_{j}},
$$

$\partial \hat{\boldsymbol{G}}_{t} / \partial \boldsymbol{U}$ is the viscous flux Jacobian, and $\boldsymbol{I}$ is the identity matrix. Following Batten et al. (1996), (33) can be reduced to a set of $N_{s}+3$ tridiagonal systems of equations which can be solved individually in order, providing account is taken of the coupling between the equations through the boundary conditions, specifically the catalytic wall conditions.

The other terms in (31) can be handled in several ways. The simplest, and most efficient computationally, is to treat them explicitly and add their contribution to the right-hand side of (33). This implicit/explicit method works well provided that the grid step in the streamwise direction is much larger than that in the transverse direction. Otherwise the stability of the scheme will be governed by the explicit treatment of the streamwise diffusion terms, which could require a time step several orders of magnitude smaller than that required by the usual Courant-type condition from the convective terms. Hence this scheme is suitable for a thin-layer type of flow in which the development of the flow is much slower in the streamwise direction than the transverse direction. For most of the calculations performed here this implicit/explicit approach is satisfactory. In fact, as is commonly done, the streamwise terms could have been safely dropped from the calculation procedure. However, as will be seen below, with a fully catalytic wall the streamwise diffusion terms are significant near the junction, and a very fine grid is required in this region. The result is that the time step becomes too small for practical purposes. In these cases it is necessary to treat the streamwise diffusion terms implicitly, at least in part. One way of doing this is to perform linearized implicit solutions in both directions, i.e. to include an extra step similar to (33) with $y$ replaced by $x$ and $\boldsymbol{G}_{t}$ replaced by $\boldsymbol{F}_{s}$ where $\boldsymbol{F}_{s}$ contains derivatives in $x$ only. Another way of stabilizing the numerical method to an extent, without introducing an additional solution step, is to treat the central terms in the streamwise diffusion operator implicitly, i.e. add these terms to the right-hand side of (33), and include the effects of the central terms (those involving elements of $\boldsymbol{U}_{j}$ ) on 
the left by augmenting the Jacobian. For this semi-implicit method, which is similar to the classic leapfrog method, the time step required with the very fine grid used for the fully catalytic case is an order of magnitude smaller than for an implicit scheme in both directions, but at least an order of magnitude larger than for the basic method when the streamwise terms are treated explicitly. However, it has an important advantage over the scheme with a full implicit solution in the streamwise direction in that it uses only local information, and can therefore be implemented in the parallel code with no further communications overheads. In contrast, the scheme with the full implicit solve couples all grid points along a line in the streamwise direction, so that there would be either a drastic increase in communications, or a significant increase in the complexity of the code to implement this scheme in parallel. The results presented below for the fully catalytic wall were produced using the semi-implicit method.

The cross-derivative viscous terms, when included, were treated explicitly, and were added for example to the right-hand side of (33). These terms, however, could have been safely dropped. Even with the fully catalytic wall where the streamwise diffusive effects were important, tests showed that including the cross-derivative terms does not significantly affect the results.

Although there are three species, only one of them, the atomic oxygen fraction $Y_{O}$ need be calculated directly in the viscous step. Argon serves only as a collision partner in the reaction scheme, hence its mass fraction will not change, and once the change in $Y_{O}$ has been determined, the change in $Y_{O_{2}}$ is known.

Due to possible stiffness in the reaction scheme, an implicit method is required for the reactive contribution. Equation (32) can be linearized to give

$$
\left[\boldsymbol{I}-\Delta t\left(\frac{\partial \dot{\boldsymbol{\omega}}}{\partial \boldsymbol{U}}\right)^{n}\right] \delta \boldsymbol{U}=\Delta t \dot{\boldsymbol{\omega}}^{n} .
$$

This relation is similar to that for the viscous solver (33), although since the source terms $\dot{\omega}$ do not involve spatial derivatives, (35) can be solved at each point independently. It is assumed here that the reaction-induced changes over time $\Delta t$ are small enough that this linearization can be used. For the particular reaction scheme used here, this assumption is valid. In other cases, however, this may not be true. For example, with a reactive air system (Amaratunga 1998) it was necessary to use a full nonlinear implicit method. This is easily achieved by converting (35) to an iterative Newton-Raphson scheme by updating the Jacobian and the right-hand side during each iteration.

There are a number of possible ways of updating the overall solution from the solutions from the separate operators. A standard way is to use a symmetric sequence of the form

$$
\boldsymbol{U}^{n+2}=L_{x} L_{y} L^{V} R R L^{V} L_{y} L_{x} \boldsymbol{U}^{n},
$$

where $L_{x}$ and $L_{y}$ are the inviscid operators in the $x$ - and $y$-directions respectively, $L^{V}$ is the viscous operator and $R$ the reactive operator. In this scheme the vector of conserved variables is updated after each substep. This requires the temperature to be calculated iteratively eight times at each grid point to advance the solution by $2 \Delta t$. Calculating $T$ from (12) is a relatively expensive part of the complete calculation scheme, and with this symmetric sequence of updates, it can consume more than half the total computational time. Accordingly, a simpler and quicker update sequence was also tried. Here the contribution from the convective $\left(\delta \boldsymbol{U}_{C x}\right.$ and $\left.\delta \boldsymbol{U}_{C y}\right)$, viscous $\left(\delta \boldsymbol{U}_{V}\right)$ and reactive $\left(\delta \boldsymbol{U}_{R}\right)$ operators were calculated separately using $\boldsymbol{U}^{n}$ as the current state, 


$\begin{array}{cccc}\text { Grid } & \text { CFL } & q_{w} & Y_{O} \\ 50 \times 50 & 1.30 & 1.347 \times 10^{6} & 0.0916 \\ 50 \times 100 & 1.30 & 1.328 \times 10^{6} & 0.0903 \\ 98 \times 50 & 1.30 & 1.350 \times 10^{6} & 0.0917 \\ 98 \times 100 & 1.30 & 1.332 \times 10^{6} & 0.0905 \\ 98 \times 200 & 1.30 & 1.323 \times 10^{6} & 0.0900 \\ \text { TABLE 6. The surface heat transfer rate } q_{w} \text { and atomic oxygen mass fraction } Y_{O} \text { at } x / L=1.8 \mathrm{~cm} \\ \text { for different grids with non-catalytic case 1. The chemistry is KD:PM. }\end{array}$

and the variables then updated using

$$
\boldsymbol{U}^{n+1}=\boldsymbol{U}^{n}+\delta \boldsymbol{U}_{C x}+\delta \boldsymbol{U}_{C y}+\delta \boldsymbol{U}_{V}+\delta \boldsymbol{U}_{R},
$$

which requires only two temperature calculations to advance the solution by $2 \Delta t$. Both sequences were tried and no significant differences were found. Hence, the cheaper, non-symmetric sequence (37) was used for the results presented below, except in the case when the wall was fully catalytic, when the symmetric sequence was used, although more for reasons of numerical stability than accuracy. We note, however, that this sequence may not always be suitable. In particular, it was found that in certain cases with a reactive air scheme the tighter coupling in the symmetric scheme (36) was required (Amaratunga 1998).

The time step for the scheme is dictated by the stability requirements of the explicit inviscid solver. An artificial Courant number of 1.3, based on the minimum cell length and the maximum wave speed returned by the Riemann solver, was used to set the time step for most of the runs. The exception to this was with the fine grid runs for the fully catalytic case, when the Courant number was set to 0.1 with the semi-implicit scheme discussed above. Taking a smaller time step with a fixed grid had a smaller effect than varying the grid (see table 6 below), so for any particular run, a time step close to the maximum possible was used.

Free-stream values are used for the initial conditions, which implies an instantaneous introduction of the plate into the moving fluid. This required a small step initially, with the artificial Courant number set at 0.25 , but this could be increased after a few hundred time steps. The procedure was time marched to a steady state, with the convergence criterion being that the $L_{2}$ measure of the change in the density over a complete time step should be less than $10^{-8}$. When starting from the free-stream conditions, convergence was achieved within a dimensionless time of 16 .

The grid used in the calculations is a stretched Cartesian grid, with grid points clustered in $x$ near the leading edge of the plate, and near the surface in $y$. The clustering was achieved using a transformation

$$
\mathrm{d} z_{i}=\frac{\mathrm{d} \eta}{1+\alpha \exp \left(-\beta \eta_{i}^{2}\right)}, \quad \eta_{i}=i \mathrm{~d} \eta, \quad z_{0}=0, \quad z_{i}=z_{i-1}+\mathrm{d} z_{i}
$$

in both directions. The grid in $z$ was then rescaled so that the maximum value of $x$ was 2.5 , and that of $y$ approximately 1.5 . For most of the runs reported below, in the $x$-direction $\alpha=1$ and $\beta=19$ were used, and in $y, \alpha=2500$ and $\beta=33$. The grid step in $y$ near the surface is very small. This was necessary in order to adequately resolve the chemical effects near the surface. Without the chemistry, the grid step near the surface could be an order of magnitude larger: $\alpha=250$ and $\beta=22$ were found to be sufficient with chemically frozen flow even with catalytic surface effects. 
Two of the key variables in this study are the mass fraction of atomic oxygen, $Y_{O}$ and the dimensional surface heat transfer rate, $q_{w}$ at the junction, $x=1.8 \mathrm{~cm}$. Table 6 gives these values for a number of different grids for case 1 with KD:PM for the chemistry and a non-catalytic surface. From table 6 it can be seen that provided there are at least 100 points in $y$, the relative change in the solution is less than $1 \%$, while the $50 \times 50$ grid will provide acceptable accuracy for checking the general behaviour of the solution, for example with the different chemical schemes. Unless otherwise mentioned below, the $98 \times 100$ grid has been used.

Although satisfactory convergence has been obtained, the accuracy of this solution is not guaranteed. The non-catalytic surface heat transfer rate profile was checked by comparing the numerical solution with that produced by the empirical Eckert formula (Eckert 1955) for the surface heat flux for an isothermal flat plate. Excellent agreement was obtained, and the empirical and numerical heat fluxes were graphically indistinguishable on the scale of figure 2 below. Further, excellent agreement has been obtained with experimental results for flat-plate flows for Mach numbers up to 6.85 (Amaratunga 1998).

\section{Chemically frozen flow}

For reference, and to examine the basic effects of the surface catalysis, first we consider flow in which there are no chemical reactions in the gas phase: specifically, case 1 with $K_{f}=K_{b}=0$ so that the change in the composition of the gas mixture is forced explicitly by the surface chemistry.

The surface heating rate, which is one of the major variables in this problem, is given, with a change in sign, by $q_{y}$ from (8) and (9) at $y=0$. It can be split into thermal $\left(q_{w_{T}}\right)$ and catalytic $\left(q_{w_{C}}\right)$ contributions, given by

$$
\begin{gathered}
q_{w_{T}}=\left(\lambda \frac{\partial T}{\partial y}\right)_{w}, \\
q_{w_{C}}=\left(\mu \frac{L e}{P r} \sum_{s=1}^{N_{s}} h_{s} \frac{\partial Y_{s}}{\partial y}\right)_{w} .
\end{gathered}
$$

Figure 2 shows $q_{w_{T}}$ and $q_{w_{C}}$ when $\gamma_{w}=10^{-2}$, and $q_{w}=q_{w_{T}}$ for the non-catalytic $\left(\gamma_{w}=0\right)$ case. In this case, on the scale used in figure 2 , the thermal heat flux for the catalytic case is almost indistinguishable from the surface heat flux of the noncatalytic plate. Also, the heating rate changes discontinuously over a single grid cell at the catalytic junction, which suggests no significant upstream influence from the catalytic region. Examination of the species mass fractions at the wall confirms that this is indeed the case, with $Y_{O}=0.1258$ at $x=1.8 \mathrm{~cm}$ but the same as the specified incoming value of 0.142 to three significant figures at the grid point before. Figure 2 has as its horizontal axis $x / L$ where $L$ is the reference length of $1 \mathrm{~cm}$. All figures presented below will use this reference length. The vertical axis gives the heat transfer rate in $\mathrm{W} \mathrm{m}^{-2}$. All values for heat transfer below will use these units or $10^{6} \mathrm{~W} \mathrm{~m}^{-2}$ (which should be clear from the context) and, where convenient, the units will be dropped henceforth.

Closer inspection of the thermal heat flux shows that there is some change due to the catalysis but on too small a scale to be seen in figure 2. In fact there are two counteracting changes in the solution. The surface reaction is exothermic, and consequently the fluid near the wall is heated, leading to an increase in the 


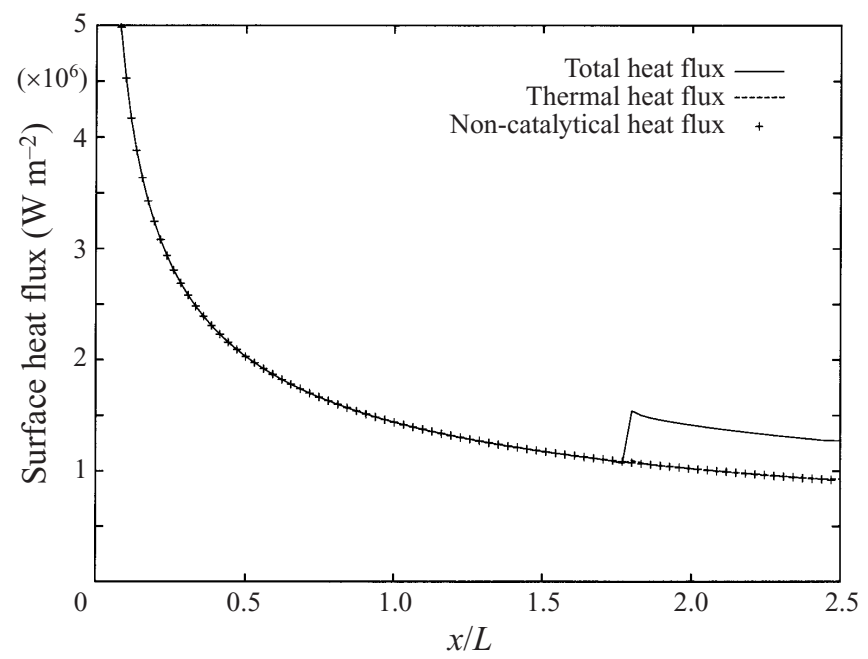

FIGURE 2. Total and thermal surface heat transfer rate solution for case 1 with frozen chemistry and the non-catalytic heat transfer rate solution for the same conditions. $L$ is a reference length of $1 \mathrm{~cm}$.

temperature gradient near the wall. In contrast, due to the change in composition of the gas mixture, the thermal conductivity decreases, but by less than $5 \%$ by $x / L=2.5$. These two effects are opposed, but do not cancel each other. The result is a small decrease in the thermal heat flux due to the catalysis. However, this change is an order of magnitude less than the decrease which occurs in this region because of the continuous growth of the boundary layer, which both the catalytic and non-catalytic solutions display. Hence the apparent lack of change in $q_{w_{T}}$ in figure 2 in the catalytic region.

The observation that there is very little change in the thermal component of the surface heat flux may not extend to other, more general, cases. In particular, as will be seen below, there is a much larger difference between the thermal heat transfer rate in catalytic and non-catalytic cases when the boundary layer is not chemically frozen. Also, this agreement may depend on the details of the modelling. A test calculation was performed with $\lambda$ frozen at its free-stream value, and a significant change in $q_{w_{T}}$ was observed in the catalytic region.

The boundary condition for the mass fraction of $\mathrm{O}$ and $\mathrm{O}_{2}$ is discontinuous at the catalytic junction. As a result, while the mass fractions are continuous there, their slope is infinite. A self-similar analytical model can be derived for the chemical composition in the neighbourhood of the junction which gives the nature of the singularity at the junction. For generality consider the dissociation/recombination reaction mechanism

$$
X_{2}+M \rightleftharpoons X+X+M,
$$

where $M$ is $X_{2}, X$, or $N$, and $N$ acts only as a collision partner. Assume that near the catalytic junction, very close to the surface the temperature, the total density, and all transport coefficients are constant to leading order. Assume also that the flow is given by a simple linear shear flow, so that $u=\alpha y, v=0$ where $\alpha$ is a constant. The species density equation for the atomic component $X$ becomes

$$
\rho \alpha y \frac{\partial Y_{X}}{\partial x}=\frac{\mu L e}{P r} \frac{\partial^{2} Y_{X}}{\partial y^{2}}+\dot{w}_{X}
$$


where for generality the chemical effects have been retained. The mass fraction of $X_{2}$ can be written as

$$
Y_{X_{2}}=1-Y_{N}-Y_{X},
$$

where $Y_{N}$ is constant. With constant density and temperature the source term for $X$ can be written as

$$
\dot{w}_{X}=a+b Y_{X}+c Y_{X}^{2}+d Y_{X}^{3}
$$

where $a, b, c$ and $d$ are constants. The thin-layer equation (42) now becomes

$$
\rho \alpha y \frac{\partial Y_{X}}{\partial x}=\frac{\mu L e}{P r} \frac{\partial^{2} Y_{X}}{\partial y^{2}}+a+b Y_{X}+c Y_{X}^{2}+d Y_{X}^{3} .
$$

The boundary conditions are

$$
\left.\begin{array}{ll}
\frac{\partial Y_{X}}{\partial y}=0, & x-x_{0}<0, \\
\frac{\partial Y_{X}}{\partial y}=\gamma Y_{X}, & x-x_{0}>0,
\end{array}\right\}
$$

where the catalytic junction is at $x=x_{0}$ and

$$
\gamma=\gamma_{w}\left(\frac{R_{0} T_{w}}{2 \pi M_{X}}\right)^{1 / 2}\left(\frac{\rho P r}{\mu L e}\right)_{w}
$$

from (20). Assuming that the mass fraction of the atomic species at $x_{0}$ is given by $Y_{X}=Y_{X J}$ and that there is a continuous change in $Y_{X}$ at $x_{0}$ which does not necessarily display a regular behaviour, we write

$$
Y_{X}=Y_{X J}+\eta^{n} f(\zeta)+\cdots,
$$

where $\eta=x-x_{0}, \zeta=y / \eta^{n}$ is of order one and $n$ describes the leading behaviour of $Y_{O}$ at the surface. Equation (45) now becomes

$$
\rho \alpha \eta^{n} \zeta\left[n \eta^{n-1} f(\zeta)-n \eta^{n-1} \zeta f^{\prime}(\zeta)\right]=\frac{\mu L e}{P r} \eta^{-n} f^{\prime \prime}(\zeta)+a+b Y_{X J}+c Y_{X J}^{2}+d Y_{X J}^{3}+O\left(\eta^{n}\right) .
$$

Equating the order of $\eta$ on the left-hand side to the leading order of $\eta$ on the right-hand side gives

$$
n=\frac{1}{3} \text {. }
$$

From (49) it can be seen that the leading behaviour of the atomic oxygen composition is $\eta^{1 / 3}$ irrespective of the reaction mechanism, since the dominant terms will always be the convective and diffusive terms. Introducing the scalings

$$
f(\zeta)=\kappa g(z), \quad \zeta=l z
$$

where

$$
\kappa=\gamma l Y_{X J} \quad \text { and } \quad l=\left(\frac{3 \mu L e}{\rho \alpha P r}\right)_{w}^{1 / 3},
$$

the governing equation for the self-similar solution for $x-x_{0}>0$ is

$$
g^{\prime \prime}+z^{2} g^{\prime}-z g=0
$$

and the boundary conditions are

$$
g^{\prime}(0)=1 \quad \text { and } \quad g \rightarrow 0 \quad \text { as } \quad z \rightarrow \infty .
$$




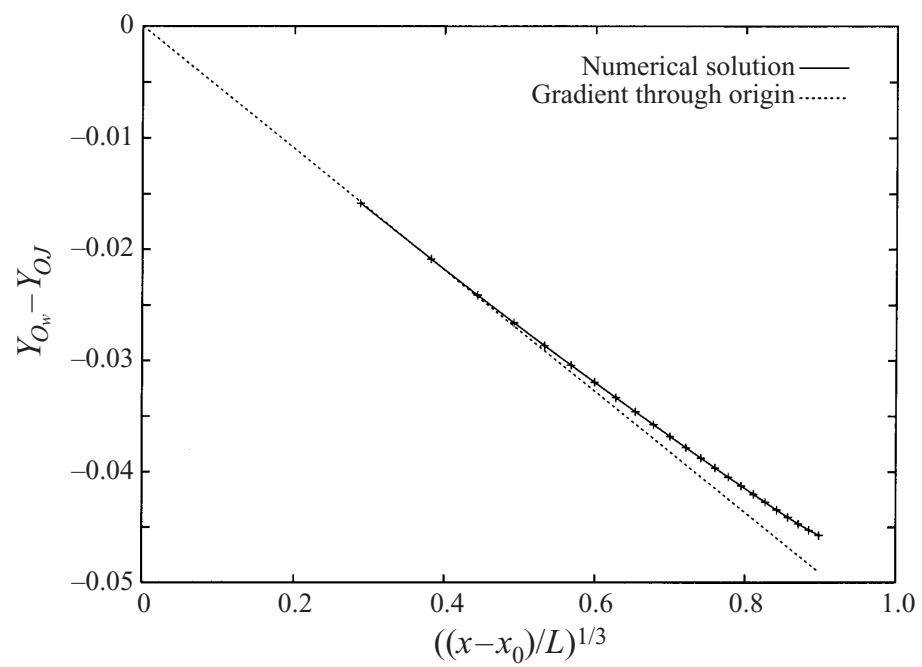

FIGURE 3. Plot of $\left(Y_{O_{w}}-Y_{O J}\right)$ against $\left(\left(x-x_{0}\right) / L\right)^{1 / 3}$ for the numerical surface distribution of atomic oxygen in the discontinuous region.

The problem defined by (53) and (54) is easily solved numerically. The solution shows a simple monotonic increase from $g=-1.065$ at $z=0$ to the far-field value of zero, which in practice is obtained before $z$ reaches 5 .

On the surface for $x>x_{0}$, the atomic species mass fraction is given by

$$
Y_{X_{w}}=Y_{X J}-1.065 \kappa\left(x-x_{0}\right)^{1 / 3}
$$

where $Y_{X J}$ is taken to be the free-stream value for $Y_{X}$.

For the specific case considered here, the predicted values for $Y_{O}$ from (55) are compared with the values from the numerical solution in figures 3 and 4 . Numerically, the first point with catalytic behaviour lies at $x / L=1.8$ and the point immediately upstream is non-catalytic. Hence, in practice the transition from non-catalytic to catalytic behaviour at the surface occurs at some intermediate location between these two points which is not determined exactly by the numerical method. Accordingly, the value of $x_{0}$ used in plotting figures 3 and 4 was found by adjusting $x_{0}$ until the plot of $\left(Y_{O w}-Y_{O J}\right)$ against $\left(x-x_{0}\right)^{1 / 3},\left(x>x_{0}\right)$, gave a straight line through the origin in the leading region. The value used was $x_{0} / L=1.776$, which is located at 0.75 of the grid step immediately upstream of $x / L=1.8$.

As can be seen from figures 3 and 4 there is excellent agreement between the numerical and analytical predictions of the change in the mass fraction immediately downstream of the catalytic junction, with, not surprisingly, a gradual deviation between the numerical and analytical values further downstream. The agreement found here is extremely good considering the relative coarseness of the grid in the region of the discontinuity in the boundary conditions. The grid step in $x$ at $x / L=1.8$ is $0.032 L$, and from $x=x_{0}$ to $x / L=1.8$ the predicted drop in the value of $Y_{O}$ is 0.016 , which is more than $10 \%$ of its incoming value.

From (20) and (40), the chemical contribution to the surface heat flux is

$$
q_{w_{C}}=\gamma_{w} \sqrt{\frac{R_{0} T_{w}}{2 \pi M_{O}}} \rho_{w} Y_{O_{w}}\left(h_{O_{w}}-h_{O_{2 w}}\right) .
$$

With (55) and values from the non-catalytic solution (the specified wall temperature 


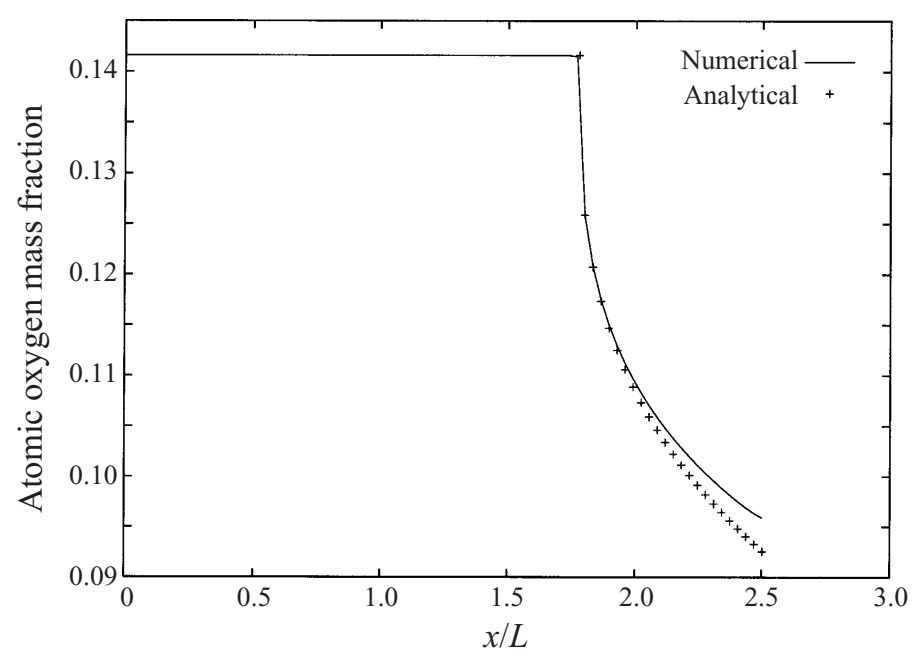

FIgURE 4. Numerical surface distribution of atomic oxygen and the analytical solution in the discontinuous region.

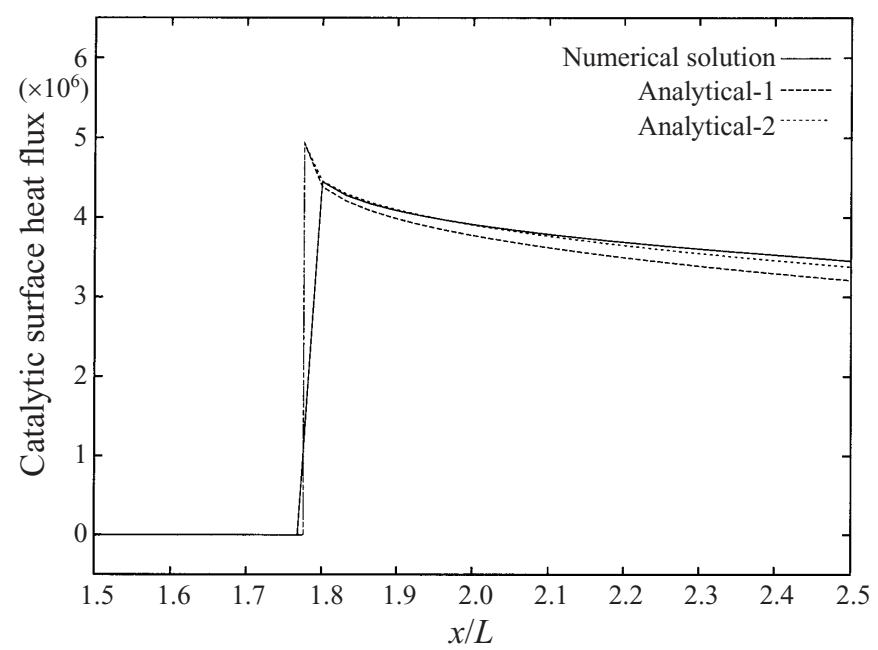

FIGURE 5. Numerical and theoretical catalytic surface heat fluxes. The solid line is the numerical solution, the dashed line uses the density from the non-catalytic solution and the dotted line allows for the change in density due to the change in composition of the gas.

and the numerical value of $\rho_{w}$ ), (56) can be used to estimate the catalytic contribution to $q_{w}$, at and downstream of the junction. Figure 5 shows the distribution of $q_{w_{C}}$ along the surface using the same value for $x_{0}$ as above. The numerical value of $q_{w_{C}}$ at $x / L=1.8,4.448 \times 10^{5}$, is slightly greater than that predicted from (55) and (56), $4.384 \times 10^{5}$, and, as expected, both are significantly lower than the predicted value of $4.933 \times 10^{5}$ at $x=x_{0}$ because of the rapid change in $Y_{O}$ near the junction and the coarseness of the grid there. As can be seen from figure 5, the predicted values of $q_{w_{C}}$ are consistently lower than the numerical ones.

However, the prediction can be improved by taking account of the change in the fluid density at the wall in the catalytic region. Above, in developing the self-similar solution for $Y_{O}$ near the catalytic junction, it was assumed that the density was 
constant. However, inspection of the numerical solution with catalysis shows that the density varies from that from the non-catalytic solution. This would be expected because of the rapid change in composition of the gas in this region: for a flow past a flat plate, or indeed any attached thin-layer flow, the change in pressure across the boundary layer from the free-stream value would be expected to be relatively small, but since a change in composition in the mass fraction of the gas will generate a change in the mixture gas constant $R$, there should be a corresponding change in the total density of the gas at the wall. Inspection of the catalytic and noncatalytic solutions showed that while there is a difference in the surface pressures, proportionally it is an order of magnitude smaller that the change in density, and therefore, since the wall temperature is the same, most of the change in density must come from the change in composition of the gas, which produces a decrease in $R$ and an increase in $\rho$. Hence, by assuming the pressure is the same as in the non-catalytic case, and using (55) to calculate the surface distribution of $Y_{O}$ and $Y_{O_{2}}, \rho$ can be estimated from the equation of state (11). This calculation has been performed, and, as shown in figure 5, it removes most of the difference between the predicted and numerical catalytic surface heat flux.

As the grid is refined, the effective value for $x_{0}$ should approach $1.8 \mathrm{~L}$, and the numerical value of $q_{w_{C}}$ at $x=1.8 \mathrm{~L}$ should approach the predicted value, but should not reach it, since in a small region very close to the junction the streamwise diffusion terms, which are not taken into account in the model, will be significant and will smooth out the abrupt change assumed above. A calculation with a much finer grid near the junction $\left(\Delta x / L \approx 2.6 \times 10^{-5}\right)$ gave $Y_{O}=0.1384$ at $x=1.8 L$, a $2 \%$ drop from the free-stream value, and $q_{w_{C}}=4.846 \times 10^{5}$, again $2 \%$ below the predicted value. For comparison, the coarse $50 \times 50$ grid gave $q_{w_{C}}=4.32 \times 10^{5}$. Note that although the fine-grid calculation gives better resolution near the junction, it is much more expensive computationally than that for the standard $98 \times 100$ grid. The results from the fine grid could have been used for figures 2 to 5 , but the standard grid was used instead as it is the one used for most of the calculations/cases, and to demonstrate the quality of the results that can be obtained near the junction with a relatively coarse grid.

So far we have considered non-catalytic and partially catalytic surfaces. Assume now that the surface downstream of the junction is fully catalytic with $\gamma_{w}=1$. The theory presented above predicts that $q_{w_{C}}$ at the junction will be 100 times larger than that with $\gamma_{w}=10^{-2}$, i.e. $4.933 \times 10^{7}$, which far exceeds the thermal component obtained from the non-catalytic solution of $1.076 \times 10^{6}$. Also, from (55), the estimated distance for $Y_{O}$ to drop from its free-stream value of 0.1416 to zero is $1.75 \times 10^{-5} \mathrm{~L}$, which is much less than the grid step near the junction for the $98 \times 100$ grid of $3.2 \times 10^{-2} \mathrm{~L}$. Hence, a much finer grid is needed in this case, and it appears likely that the streamwise diffusion term will play a significant role. The atomic oxygen mass on the surface near the junction is shown in figure 6 , and it is clear that there is a significant amount of upstream influence. The results shown are for a grid with $\Delta x / L \approx 2.6 \times 10^{-5}$, but are graphically identical to those found with $\Delta x / L \approx 1.1 \times 10^{-6}$. Also shown in figure 6 is $Y_{O}$ for a calculation for the thin-layer case when the streamwise diffusion terms are dropped, from which can be seen clearly the upstream influence of the diffusion. The numerical solution gave $Y_{O} \approx 0.06$, and $q_{w_{C}} \approx 2.1 \times 10^{7}$ at the junction, both around half the values predicted by the model. Nevertheless, $q_{w_{C}}$ is still easily the dominant part of the surface heat flux in this region.

A simplified model of the problem near the junction is obtained by assuming that 


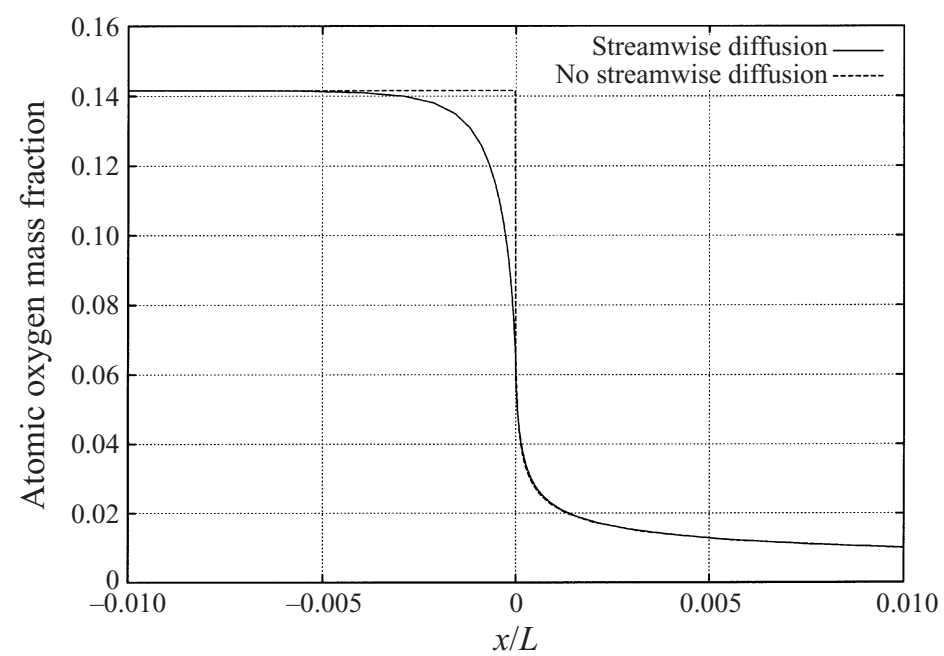

FIGURE 6. Atomic oxygen mass fraction near the junction with $\gamma_{w}=1$. The abscissa is $x / L-1.8$.

there is a simple shear flow in this region and that all transport coefficient and other parameters in the problem are constant. The problem for $Y_{O}$ then reduces to a convection-diffusion problem governed by

$$
\nabla^{2} Y=\beta y \frac{\partial Y}{\partial x}
$$

with surface conditions

$$
\left.\begin{array}{ll}
\frac{\partial Y}{\partial y}=0, & x<0, \\
\frac{\partial Y}{\partial y}=\gamma_{m} Y, & x>0,
\end{array}\right\}
$$

where $Y$ is $Y_{O}$ with its free-stream value scaled out, there is an unspecified but small scaling and a change of origin in the coordinate system, and $\beta$ and $\gamma_{m}$ are as yet unspecified generalized shear rate and catalytic efficiency respectively. The conditions used on the other boundaries are

$$
\begin{gathered}
Y=1 \quad \text { as } \quad x \rightarrow-\infty, \\
\frac{\partial^{m} Y}{\partial x^{m}} \rightarrow 0 \text { as } \quad x \rightarrow \infty,
\end{gathered}
$$

and

$$
\frac{\partial^{m} Y}{\partial y^{m}} \rightarrow 0 \quad \text { as } \quad y \rightarrow \infty,
$$

where $m>2$. The upstream condition (59) fixes $Y\left(Y_{O}\right)$ at the incoming (free-stream) value, while the other far-field conditions were chosen as they do not explicitly force a value on $Y$. In fact, numerically, the downstream boundary $x=x_{\max }$ was placed sufficiently far downstream that its exact form did not affect the results near the junction, while the position of outer boundary $y=y_{\max }$ was such that the value of $Y$ there was asymptotically close to 1 . A value of $m=4$ was used for the results presented below. We note that the inhomogeneous surface condition does not admit 


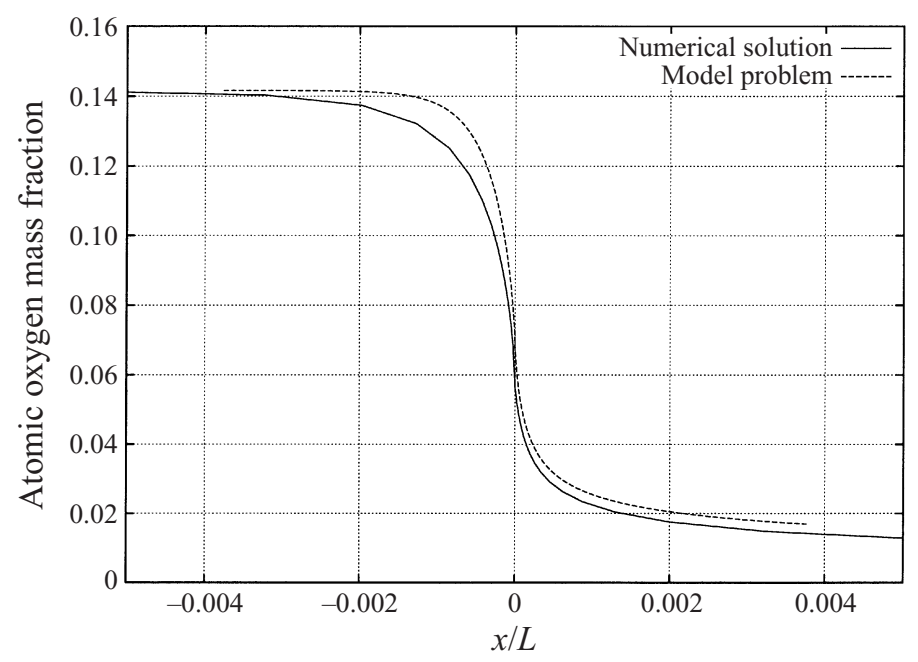

FIGURE 7. Atomic oxygen mass fraction for the full numerical solution and the analytic model solution near the junction for $\gamma_{w}=1$. The abscissa is $x / L-1.8$.

a solution in the form of a power series in the distance from the junction, which is a standard way of approaching problems of this kind.

Relating the parameters in (57)-(61) to the original governing equation for $Y_{O}$, we see that

$$
\beta=\epsilon^{2} \beta_{0}, \quad \text { where } \quad \beta_{0}=\frac{\rho \alpha P r}{\mu L e},
$$

and

$$
\gamma_{m}=\epsilon \gamma,
$$

where $\epsilon$ is the small parameter in the mapping $\left(x-x_{0}, y\right) \rightarrow \epsilon(x, y)$ between the coordinate systems.

Examination of the numerical solution shows that near the junction $\beta_{0}=O\left(10^{10}\right)$ and $\gamma=O\left(10^{5}\right)$, and hence if $\epsilon=O\left(10^{-5}\right)$, which is consistent with the results shown in figure 6 , then both $\beta$ and $\gamma_{m}$ will be $O(1)$. It follows that there will be a balance of diffusive, convective and catalytic effects near the junction. Since $\beta$ is non-zero, (57)-(61) can be reduced to a single parameter problem with the further mapping $(x, y) \rightarrow \beta^{-1 / 2}(x, y)$, setting $\beta=1$ in (57) and replacing $\gamma_{m}$ by $\gamma_{p}=\gamma_{m} \beta^{-1 / 2}$ in (59). Now

$$
\gamma_{p}=\gamma \beta_{0}^{-1 / 2}
$$

and therefore a value for $\gamma_{p}$ can be obtained directly from the numerical solution to the full problem. There is however considerable variation in the model parameters in the region of the junction as calculated from the full numerical solution: more than $20 \%$ in $\beta_{0}$ and $\gamma$ and consequently, more than $10 \%$ in $\gamma_{p}$. The value used, $\gamma_{p}=1.92$, was taken from the full numerical solution at the junction. This value lies about the middle of the range of values spanned by $\gamma_{p}(1.8-2.0)$. The solution for $Y_{O}$ from the model problem (57)-(63) is compared with that from the full numerical solution in figure 7 , where the model coordinates have been scaled by $\epsilon_{0}=\beta_{0}^{-1 / 2}=3.8 \times 10^{-4} L$, using the value of $\beta_{0}$ from the full solution at the junction. The model problem overestimates $Y_{O}$. This is not surprising since $\beta_{0}$ increases rapidly through the junction, and therefore using a value fixed at the junction in this model 


\begin{tabular}{|c|c|c|c|c|c|c|c|}
\hline \multirow[b]{2}{*}{ Grid } & \multirow[b]{2}{*}{$K_{f}: K_{b}$} & \multirow[b]{2}{*}{$q_{w_{N}}$} & \multicolumn{2}{|c|}{ Case 5} & \multicolumn{2}{|c|}{ Case 9} & \multirow[b]{2}{*}{$Y_{O}$} \\
\hline & & & $q_{w_{E}}$ & $\operatorname{diff}(\%)$ & $q_{w_{E}}$ & $\operatorname{diff}(\%)$ & \\
\hline $50 \times 50$ & $\mathrm{KD}: \mathrm{PM}$ & 1.446 & 1.382 & 4.6 & 1.465 & -1.3 & 0.0924 \\
\hline $98 \times 100$ & $\mathrm{KD}: \mathrm{PM}$ & 1.429 & 1.382 & 3.4 & 1.465 & -2.5 & 0.0912 \\
\hline $50 \times 50$ & KD:C1 & 1.222 & 1.382 & -11.6 & 1.465 & -16.6 & 0.1367 \\
\hline $50 \times 50$ & $\mathrm{P}: \mathrm{C} 1$ & 1.206 & 1.382 & -12.8 & 1.465 & -17.7 & 0.1392 \\
\hline $50 \times 50$ & PM:C2 & 1.203 & 1.382 & -12.9 & 1.465 & -17.9 & 0.1395 \\
\hline $50 \times 50$ & $\mathrm{P}: \mathrm{P}$ & 1.811 & 1.382 & 31.0 & 1.465 & 23.6 & 0.0000 \\
\hline $50 \times 50$ & $\mathrm{P}: \mathrm{PM}$ & 1.520 & 1.382 & 10.0 & 1.465 & 3.8 & 0.0718 \\
\hline $50 \times 50$ & $\mathrm{H}: \mathrm{H}$ & 1.170 & 1.382 & -15.3 & 1.465 & -20.1 & 0.1446 \\
\hline $50 \times 50$ & none & 1.152 & 1.382 & -16.7 & 1.465 & -21.4 & 0.1472 \\
\hline
\end{tabular}

TABLE 7. Surface values at $x / L=1.8$ with different chemical schemes for non-catalytic cases 5 and $9 . q_{w_{N}}$ is the numerical heat transfer rate and $q_{w_{E}}$ the experimental value.

will overestimate the convective effects ahead of it, and thereby elevate $Y_{O}$ near the junction. In general, however, given the simplicity of this model, there is reasonable agreement between it and full numerical solutions, and it is clear that there is a threeway balance between convective, diffusive, and catalytic effects near the junction, with the streamwise diffusion playing a significant role, unlike the situation when $\gamma_{w}$ is small.

The agreement between the results from the model problem (57)-(63) and the full numerical solution could be improved greatly by using a different set of values for $\gamma_{p}$ and $\epsilon_{0}$ than those taken directly from the numerical values at the junction: close to the best fit is given with $\gamma_{p}=2.85$ and $\epsilon_{0}=7.7 \times 10^{-4} \mathrm{~L}$.

\section{The different kinetic schemes}

Two of the materials used for the experiments, alumina and silicon monoxide, are regarded as being essentially non-catalytic. Hence the effects of varying the forward and backward reactions rates was assessed by performing a series of calculations with a non-catalytic wall, and comparing the calculated heat transfer rates at $x / L=1.8$ with those measured in the experiments. Table 7 gives values for cases 5 and 9 , which have the same free-stream values and therefore the same numerical solution. Also given is the calculated atomic oxygen mass fraction at the junction. As expected, Park's original scheme (Park 1984) forces complete recombination of oxygen at the surface. In turn, for frozen chemistry and Park's scheme, the calculated surface heat transfer rate undershoots and overshoots the measured values of $q_{w_{T}}$ for these schemes, indicating that some, but not total, recombination in the boundary layer is required to obtain agreement between the experimental and numerical values. The scheme with the Kang \& Dunn values for the forward reaction and the modified version of the Park scheme for the backward reaction (KD:PM) gives the best comparison between the numerical and experimental results for both case 5 and 9 . The other schemes, apart from P:PM, provide too little recombination of oxygen and significantly underestimate the heat transfer rate in comparison with the experimental values. Calculations were also performed for all the other non-catalytic cases for the coarse grid with $\mathrm{KD}: \mathrm{PM}, \mathrm{KD}: \mathrm{C} 1$ and $\mathrm{P}: \mathrm{PM}$ kinetics, which are the three cases with best agreement in table 7, and bracket the experimental values. In all cases the best agreement between the experimental and numerical values was given with KD:PM 


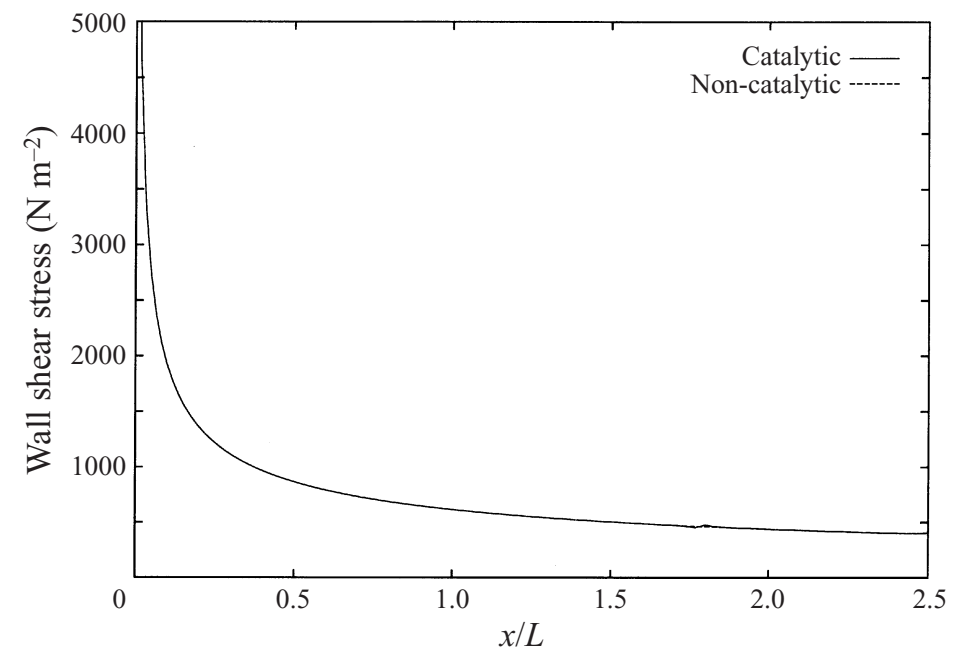

FIGURE 8 . Wall shear stress for case 1 with KD:PM kinetics. The catalytic and non-catalytic curves are almost indistinguishable on this scale.

kinetics. In fact easily the least favourable comparison for this scheme was for case 9, as given in table 7. Even allowing for the fact that a coarse grid has been used for this comparison (table 7 also includes the values for the $98 \times 100$ for KD:PM), it was clear that the KD:PM scheme gives the best agreement between the numerical and experimental results, and it has therefore been used for all the results presented below.

\section{Case 1 with chemistry}

Calculations were performed for case 1 using the KD:PM kinetic scheme and the $98 \times 100$ grid without catalysis and with catalysis with $\gamma_{w}=10^{-2}$. Plots of the shear stress, heat transfer rate, and atomic oxygen mass fraction at the wall are shown in figures 8, 9 and 10. The catalysis has no significant effect on the shear stress. As for the case with frozen chemistry, there is in effect a discontinuous change in the surface heat flux and species mass fractions at the junction, with no significant upstream influence. However, unlike in the case with frozen chemistry, there is now a noticeable change on this scale in the thermal component of the surface heat flux in the catalytic region (figure 9). Further, there are less obvious changes in the flow. The dominant chemical reactions in the gas in the boundary layer are recombination reactions (see table 4), which causes a drop in the value of the gas constant $R$. This in turn, as explained above, leads to an increase in the density. Hence, although the viscous effects will decrease the streamwise velocity $u$ near the wall, the momentum $\rho u$ may increase if the chemical reactions are vigorous enough. This happens here, and adjacent to the wall there is a thin sublayer in which the transverse velocity $v$ is negative.

There is a smooth decay in $Y_{O}$ (and corresponding growth in $Y_{O_{2}}$ ) along the wall in the non-catalytic case, but an abrupt change when the catalytic region is encountered. The self-similar model of the behaviour of the solution near the catalytic junction discussed above for the frozen chemistry case can be applied in this case as well, where $Y_{O J}$ is now the value of $Y_{O}\left(x_{0}, 0\right)$ from the non-catalytic solution. In the expansion, although the chemical effects should now be included, as demonstrated above the first 


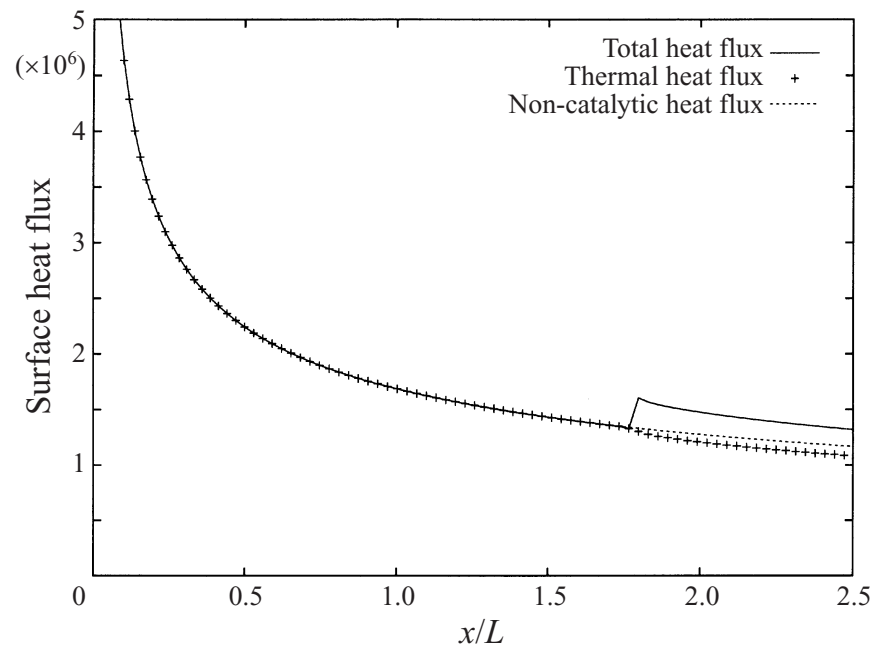

FIGURE 9. Total and thermal surface heat transfer rate for case 1 with KD:PM kinetics and surface catalysis and the non-catalytic heat transfer rate for the same conditions.

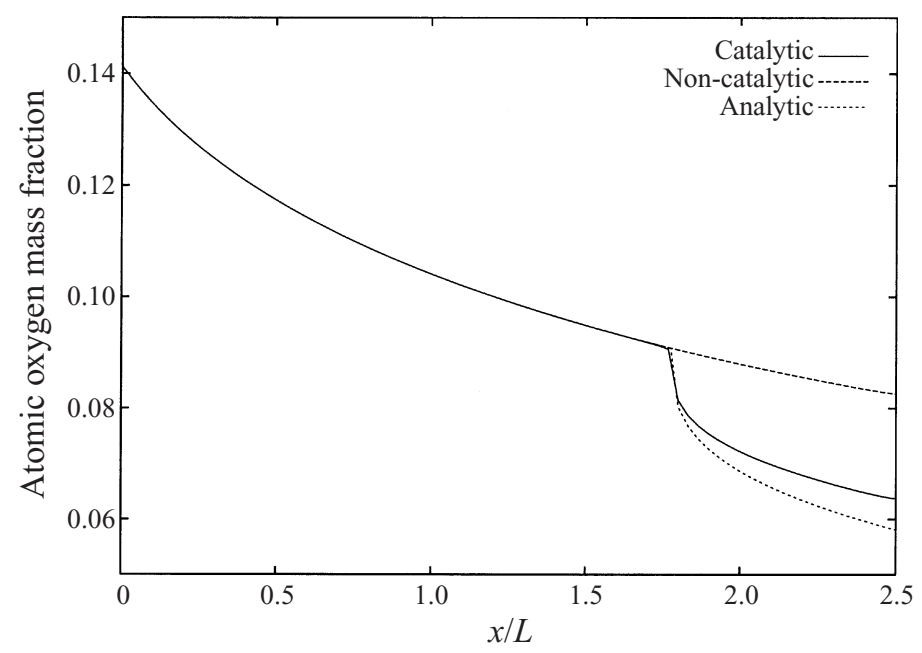

FIGURE 10. Surface atomic oxygen mass fraction for case 1 with KD:PM kinetics.

two terms in the expansion will still be given by (48) with $n=1 / 3$. However, since the base solution is no longer constant, we should not expect such a good comparison between values from the full numerical solution and the analytical solution as shown above when there are no chemical reactions.

Figure 10 shows the predicted distribution of $Y_{O w}$ from (48) as well as the catalytic and non-catalytic numerical results. Clearly, although the shape of the curves is the same, the difference between the numerical result and the analytical prediction in this case is much larger than that with frozen chemistry (figure 4). However, there is better agreement between the numerical and analytical predictions of the surface heat flux than for $Y_{O}$, as can be seen from figure 11. Again, allowing for the change in the density due to the change in composition of the gas improves the accuracy of 


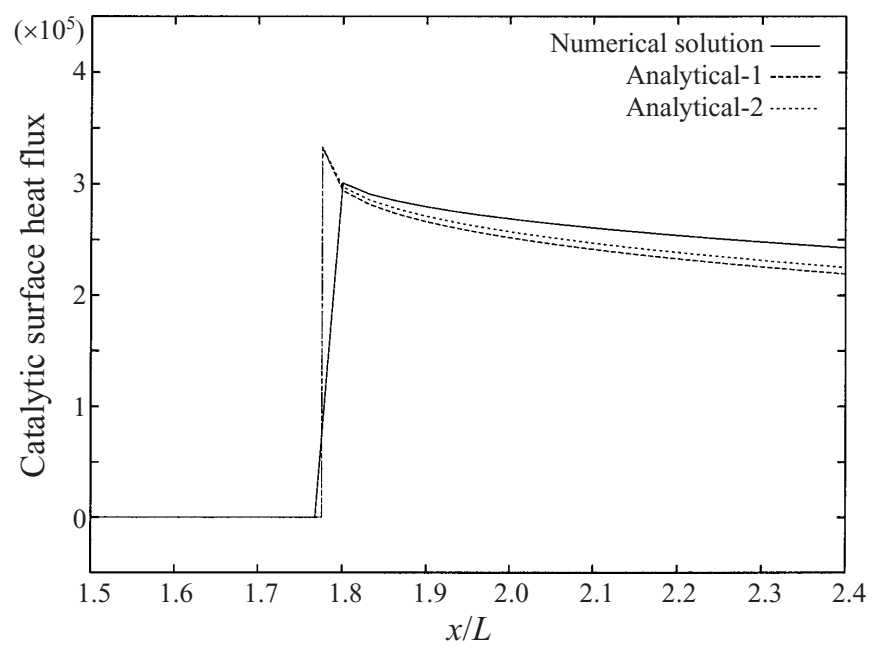

FiguRE 11. Numerical and theoretical catalytic surface heat fluxes. The solid line is from the numerical solution, the dashed line is the basic analytical prediction, and the dotted line includes the effect of the change in density.

the prediction, so that even at $x / L=2.5$ there is only $7 \%$ difference between the numerical and analytical values of $q_{w_{c}}$.

At the junction the non-catalytic solution and (56) predict that the total surface heat flux should be $q_{w}=1.663 \times 10^{6}$, from $q_{w_{T}}=1.332 \times 10^{6}$ and $q_{w_{C}}=0.331 \times 10^{6}$. For comparison, the numerical solution with a catalytic surface produces $q_{w_{T}}=1.304 \times 10^{6}$ and $q_{w_{C}}=0.301 \times 10^{6}$, giving $q_{w}=1.605 \times 10^{6}$, which again is lower than that predicted by assuming no upstream influence. The difference between the numerical and analytical values of $q_{w_{C}}$ is, proportionally, approximately the same as that found for frozen chemistry with the $98 \times 100$ grid.

The values obtained for the wall shear stress when the gas mixture is frozen are not significantly different from those shown in figure 8 for the chemically active cases. There is however a noticeable difference between the surface heat transfer rates, as shown in figure 12. Without catalysis, the heat flux is higher when there is chemical activity in the gas. Proportionally this difference increases along the plate, reaching approximately $27 \%$ by $x=2.5 \mathrm{~L}$. This increase in heat flux is due to an increase in temperature gradient at the surface due to liberation of chemical energy, even though the change in composition of the mixture leads to a drop in the thermal conductivity $\lambda$ along the plate. In the catalytic region, due to the higher value of $Y_{O}$, the catalysis has a larger effect when the gas-phase chemistry is frozen, hence the catalytic heat flux is also larger with frozen chemistry. This increase in $q_{w_{C}}$ almost compensates the decrease in the thermal heat flux, so that the total heat flux in both cases is similar in the catalytic region.

The species mass fractions of atomic and molecular oxygen across the boundary layer at two locations $(x / L=1$ and $x / L=2.4)$ for the case with $\gamma_{w}=10^{-2}$ are shown in figure 13. There is a smooth monotonic change from the surface values to the free-stream values. For the upstream non-catalytic location the pattern is similar, but with less recombination of oxygen, than in the downstream catalytic location. Note that at the surface in the non-catalytic region, the species mass fractions do in fact have zero normal derivative as required. However, the strong recombination of 


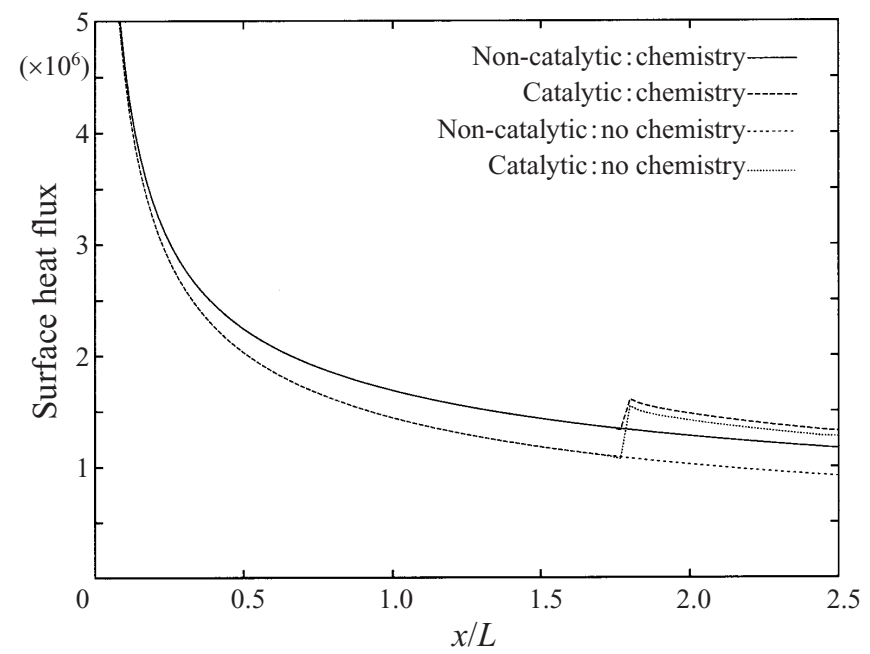

FIGURE 12 . Total surface heat flux with and without chemistry and with and without surface catalysis.

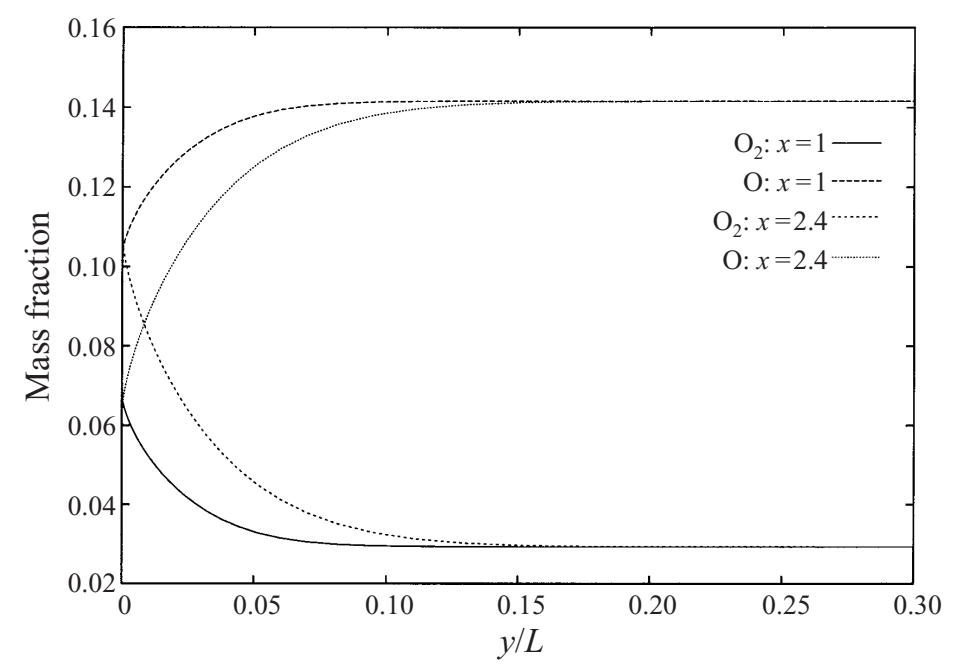

FIGURE 13. Mass fraction across the flow for case 1 with KD:PM kinetics and $\gamma_{w}=0.01$.

oxygen near the wall causes a rapid change in mass fraction in this region, leading to the apparent shape of the curve near the wall in figure 13.

With $\gamma_{w}=1$ the solution has similar behaviour to that found for the frozen chemistry case, with significant upstream diffusive effects and a value of $q_{w_{C}}$ roughly half that predicted from the results with $\gamma_{w}=10^{-2}$.

\section{Comparison with experimental results}

Calculations were performed for all the test cases given in table 5 with the KD:PM kinetic scheme, the $98 \times 100$ grid, but no surface catalysis. Table 8 gives values of the calculated surface heat flux, the percentage difference from the experimental values, and the atomic oxygen mass fraction at the position of the junction. For platinum 


$\begin{array}{cccccc}\text { Case } & \text { Material } & \begin{array}{c}\dot{q}_{w} \\ \text { experimental }\end{array} & \begin{array}{c}\dot{q}_{w} \\ \text { numerical }\end{array} & \begin{array}{c}\text { difference } \\ (\%)\end{array} & Y_{O} \\ 1 & \mathrm{Pt} & 1.675 & 1.332 & -20.5 & 0.0905 \\ 2 & \mathrm{Pt} & 2.102 & 1.442 & -31.4 & 0.0836 \\ 3 & \mathrm{Pt} & 1.759 & 1.489 & -15.4 & 0.0900 \\ 4 & \mathrm{Pt} & 1.553 & 1.159 & -25.4 & 0.0775 \\ 5 & \mathrm{Al}{ }_{2} \mathrm{O}_{3} & 1.382 & 1.429 & 3.4 & 0.0912 \\ 6 & \mathrm{SiO} & 1.436 & 1.480 & 3.1 & 0.0880 \\ 7 & \mathrm{SiO} & 1.491 & 1.457 & -2.3 & 0.0910 \\ 8 & \mathrm{SiO} & 1.553 & 1.583 & 1.9 & 0.0847 \\ 9 & \mathrm{SiO} & 1.465 & 1.429 & -2.5 & 0.0912 \\ 10 & \mathrm{SiO} & 1.541 & 1.548 & 0.4 & 0.0871 \\ 11 & \mathrm{SiO} & 1.319 & 1.377 & 4.4 & 0.0917\end{array}$

TABLE 8. Experimental and numerical surface heat transfer rates at the catalytic junction $\left(10^{6} \mathrm{~W} \mathrm{~m}^{-2}\right)$. The chemistry is $\mathrm{KD}: \mathrm{PM}$.

(cases 1 to 4 ), the calculated values of $q_{w}$ are significantly lower than the experimental values, as expected since platinum is a catalytic material. For alumina and silicon monoxide (cases 5 to 11 ), which have low catalytic efficiency, the numerical values of $q_{w}$ are scattered above and below the measured values. On average the numerical values are slightly $(1 \%)$ above the experimental ones, which is of the same order of the uncertainty in the numerical results due to grid resolution with this grid (table 6). For cases 5 and 9 , the free-stream conditions are the same, and hence the predicted value of $q_{w}$ is also the same. However, there is a $6 \%$ difference in the experimental values. If it is assumed that in both these cases the catalytic effect is negligible, then this difference can be interpreted as a measure of the experimental error. The difference between the calculated and measured values of $q_{w}$ lie within this range, and therefore it appears that the calculations are reproducing the experimental results within the uncertainties in the data.

One of the aims of the test case in the HSFF workshop (HSFF 1995) was to estimate the value of the catalytic recombination rate coefficient $\gamma_{w}$ by matching the experimental and numerical surface heat fluxes at the junction. There are two possible ways of doing this. First, the catalytic boundary conditions (20) can be implemented numerically, and the value of $\gamma_{w}$ adjusted until the fluxes match. However, as has been discussed above, in practice the change in boundary conditions will occur between the grid points, and since the peak catalytic heating rate occurs at the junction, this would overestimate the value of $\gamma_{w}$. Also, there is the basic problem of the relatively large numerical errors that will be generated in the neighbourhood of a discontinuity in the boundary conditions. An alternative, simple method for estimating $\gamma_{w}$ is to take advantage of the observation that for high-speed flows of this type there is very little upstream influence, even near the catalytic junction, and use the values from the non-catalytic solution with (56) to calculate $\gamma_{w}$ directly from the difference between the measured and thermal heat transfer rates.

Both of these methods have been used, and the results for cases 1 to 4 are given in table 9. The values obtained from the full numerical procedure are significantly larger than those found using (56). There is also a large variation in the predicted values. The values of $\gamma_{w}$ are however consistent with those found in the literature, which also show a large variation. For example, East, Stalker \& Baird (1980) use $\gamma_{w} \approx 3 \times 10^{-3}$ which they regard as typical for oxygen and nitrogen recombination on 


\begin{tabular}{|c|c|c|c|c|c|c|c|}
\hline Case & $\begin{array}{c}\dot{q}_{w} \\
\text { experimental }\end{array}$ & $\begin{array}{c}\dot{q}_{w_{T}} \\
\text { numerical }\end{array}$ & $\begin{array}{c}\dot{q}_{w_{C}} \\
\text { numerical }\end{array}$ & $\begin{array}{c}Y_{O} \\
\text { catalytic }\end{array}$ & $\begin{array}{c}\gamma_{w} \\
\text { numerical }\end{array}$ & $\begin{array}{c}\gamma_{w} \\
\text { theoretical }\end{array}$ & $\begin{array}{c}p \\
\left(\mathrm{~N} \mathrm{~m}^{-2}\right)\end{array}$ \\
\hline 1 & 1.675 & 1.297 & 0.377 & 0.0792 & $1.29 \times 10^{-2}$ & $1.04 \times 10^{-2}$ & 10531 \\
\hline 2 & 2.102 & 1.359 & 0.743 & 0.0630 & $2.76 \times 10^{-2}$ & $1.88 \times 10^{-2}$ & 11997 \\
\hline 3 & 1.759 & 1.457 & 0.302 & 0.0816 & $0.91 \times 10^{-2}$ & $0.74 \times 10^{-2}$ & 11597 \\
\hline 4 & 1.553 & 1.121 & 0.432 & 0.0640 & $1.82 \times 10^{-2}$ & $1.39 \times 10^{-2}$ & 10397 \\
\hline
\end{tabular}

TABLE 9. Heat transfer rates for platinum at the catalytic junction $\left(10^{6} \mathrm{~W} \mathrm{~m}^{-2}\right)$ and the numerical and theoretical values of $\gamma_{w}$.

oxidized metallic surfaces with a wall temperature of $300 \mathrm{~K}$. In contrast, Anderson (1973) determined values of $\gamma_{w}$ using experimental measurements of stagnation-point heat transfer rates in supersonic flow and gave a value for metallic surfaces, including platinum, of 0.09 for $400 \pm 100 \mathrm{~K}$. The standard assumption is that $\gamma_{w}$ increases with temperature, but Chen \& Chandler (1993) state, unusually, that it may also be a function of pressure. The free-stream pressures for cases 1 to 4 are included in table 9 , and clearly there is no direct relationship between $p$ and $\gamma_{w}$ in these flows.

Easily the largest value of $\gamma_{w}$ comes from case 2, which has a surprisingly large experimental heat flux. For example, compared to case 3 , the variation in the freestream conditions is less than $4 \%$, and the calculated thermal heat flux is $7 \%$ lower. In contrast, the measured heat flux is $20 \%$ higher, and the required catalytic heat flux is more than double than that for case 3 , leading to the large estimated value of $\gamma_{w}$. The difference in the measured heat flux between case 2 and cases 1,3 and 4 is more than would be expected from the change in the free-stream conditions, and the results for case 2 should be treated with caution. We note, however, that large experimental errors might be expected in this region due to the rapid change in $q_{w}$ near the junction, and that, particularly for comparison with results from numerical modelling, a distribution of measurements away from the singularity at the junction would be more satisfactory.

\section{Effect of the Lewis number}

The Lewis number, $L e$, is fixed in any particular calculation. An investigation has been performed into the effects of variations in Le. The main computations have been based on a standard value of $L e=1.4$. Calculations have also been performed for case 1 with Lewis numbers of 1.2 and 1.6 and the KD:PM kinetic scheme. The surface values of $Y_{O}$ are shown in figure 14. Due to the larger diffusive effect, the surface values of $Y_{O}$ increase with $L e$. However, although there is a noticeable difference in $Y_{O w}$ in figure 14, the change is proportionally much less than the change in Le, less than $3 \%$ in comparison with $14 \%$, respectively. There is even less change in the thermal heat flux, $q_{w_{T}}$, below $\frac{1}{2} \%$ at the site of the junction (table 10).

Also given in table 10 are the values of $\gamma_{w}$ required to match the numerical and experimental heat fluxes at the junction, obtained from the non-catalytic solution and (56). Again, the change with $L e$ is small, particularly given the uncertainties in values of $\gamma_{w}$. In general, it would appear that the assumption of constant Lewis number has had little affect on the results presented above. 


$\begin{array}{ccccc}\text { Le } & q_{w} & q_{w_{T}} & & \gamma_{w} \\ 1.2 & 1.675 & 1.326 & 0.0879 & 1.08 \times 10^{-2} \\ 1.4 & 1.675 & 1.332 & 0.0905 & 1.04 \times 10^{-2} \\ 1.6 & 1.675 & 1.334 & 0.0927 & 1.01 \times 10^{-2}\end{array}$

TABLE 10. Effect of the Lewis number: case $198 \times 100, \mathrm{KD}: \mathrm{PM}$.

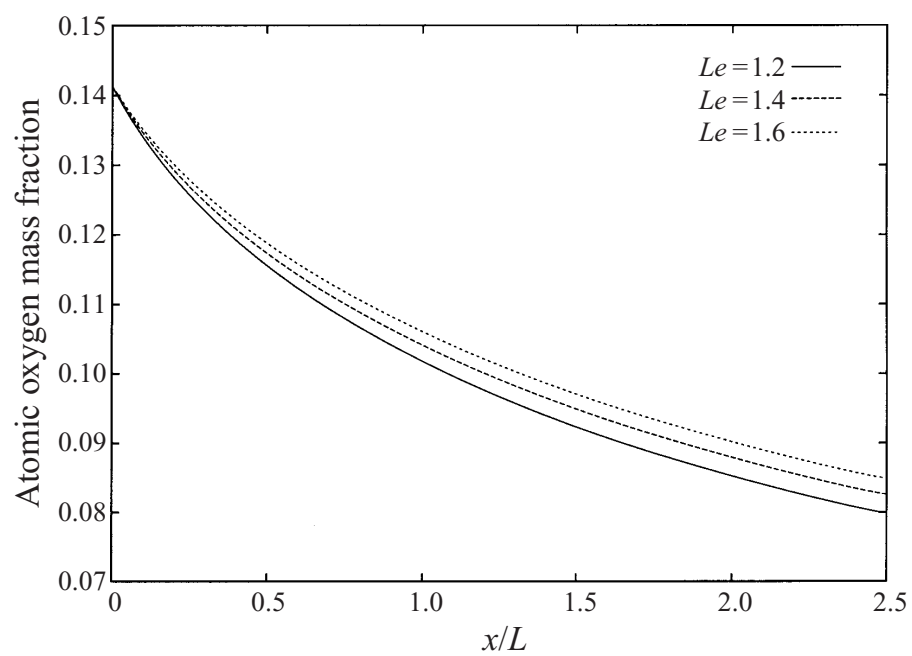

FIGURE 14. Atomic oxygen mass fractions at the surface for case 1 with $L e=1.2,1.4$ and 1.6 and KD:PM kinetics.

\section{Flow with catalytic behaviour from the leading edge}

In this paper our main interest is in the effects of discontinuous surface catalysis on the flow, in particular with change from a non-catalytic to a catalytic surface. However, situations in which the entire surface is (partially) catalytic are also of interest. A set of calculations was performed in which the surface was catalytic from the leading edge. Figure 15 shows the atomic oxygen mass fraction in the neighbourhood of the catalytic junction for case 1 with KD:PM chemistry, $\gamma_{w}=10^{-2}$, and catalysis from the leading edge and from the catalytic junction. Figure 16 shows the corresponding total and thermal surface heat fluxes. For discontinuous surface catalysis, both these figures use the fine grid referred to above with $\Delta x / L \approx 2.6 \times 10^{-5}$ near the junction.

As expected, catalysis from the leading edge leads to a significant decrease in the atomic oxygen mass fraction near the surface. Also, as before the thermal heat flux is lower than that for the non-catalytic case. However, this is more than compensated by the additional catalytic heat flux so that the total heat flux is higher than in the non-catalytic case. However, with discontinuous surface catalysis the combination of higher thermal heat flux and higher mass fraction of atomic oxygen gives a significantly higher total heat flux at $x / L=1.8$ than when there is catalysis from the leading edge. In fact, $q_{W_{C}}$ is also higher at $x / L=1.8$ with non-catalytic upstream behaviour.

With frozen gas-phase chemistry, as before, catalysis produces little change in the thermal heat flux, and hence again the total heat flux is larger when there is catalysis 


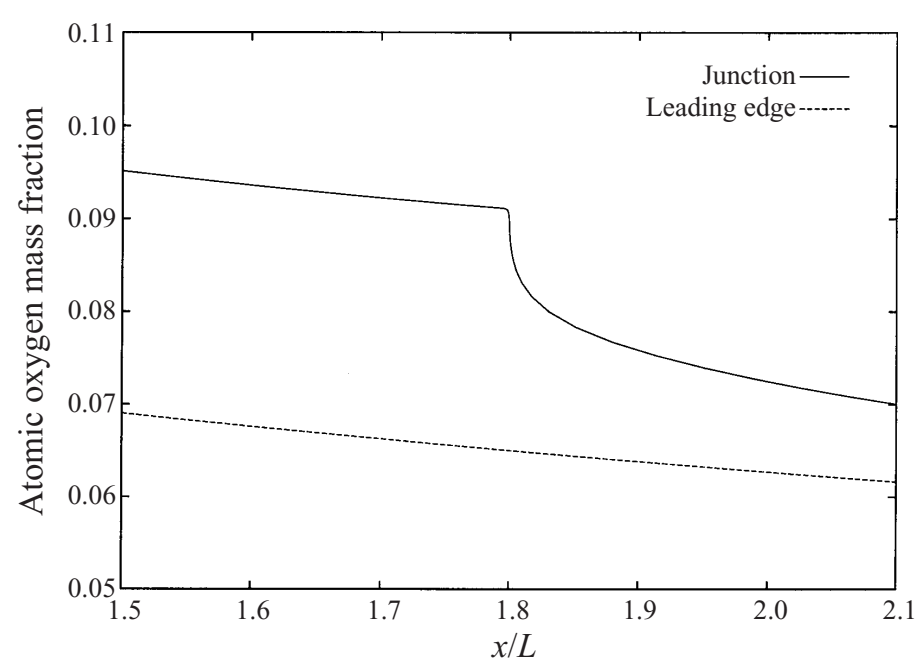

FIGURE 15. Atomic oxygen mass fractions at the surface for case 1 with catalytic behaviour from the leading edge and $x / L=1.8$. The kinetic scheme is $\mathrm{KD}: \mathrm{PM}$ and $\gamma_{w}=10^{-2}$.

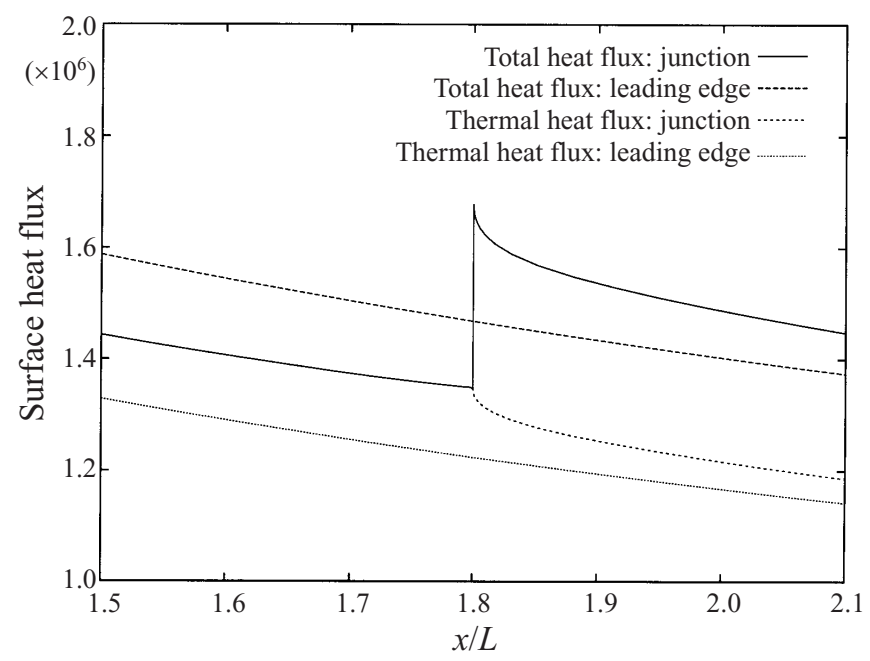

FIGURE 16. Surface heat transfer rates for case 1 with catalytic behaviour from the leading edge and $x / L=1.8$. The kinetic scheme is KD:PM and $\gamma_{w}=10^{-2}$.

from the leading edge than with a non-catalytic surface. However, again as with gasphase chemistry, with discontinuous catalysis $q_{W_{C}}$ is higher at the junction leading to a higher total heat flux there than with continuous catalysis.

These effects are magnified as the catalytic efficiency increases. Higher values of $\gamma_{w}$ lead to more depletion of atomic oxygen along the surface. Hence, while (56) indicates that increasing $\gamma_{w}$ will increase $q_{W_{C}}$, it also shows that this will be compensated, at least in part by the decrease in $Y_{O}$. In fact, for runs with $\gamma_{w}=10^{-1}$ and 1 and catalysis from the leading edge, $Y_{O}$ scaled almost inversely with $\gamma_{w}$ and hence produced very similar heat fluxes with $q_{W} \approx 1.6 \times 10^{6}$, and $q_{W_{C}}$ at $x / L=1.8$ only approximately double that for $\gamma_{w}=10^{-2}$. Further, with $\gamma_{w}=1$ and catalysis from the leading edge, the decrease in atomic oxygen near the surface due to the catalysis is much more 
important than that due to the gas-phase reactions, so that there is little difference at the wall in the solutions with and without gas-phase chemistry. Hence the surface heat flux at $x / L=1.8$ is approximately the same both with and without gas-phase chemistry. In contrast, with discontinuous surface catalysis, the atomic oxygen mass fraction is significantly greater in the neighbourhood of the junction, leading to much larger predicted heat fluxes of $2.2 \times 10^{7}$ without gas-phase chemistry and $1.5 \times 10^{7}$ with chemistry when $\gamma_{w}=1$.

\section{Concluding remarks}

A set of detailed numerical simulations have been performed for high-speed flow of a reacting gas mixture over a flat plate with discontinuous surface catalysis. Although a specific gas mixture, molecular and atomic oxygen in argon, was used for the calculations, a full range of chemical effects, both in the gas phase and at the surface, were considered, so that the results are more generally applicable to the case of dissociation and recombination of a diatomic gas in an inert diluent under a variety of conditions.

A simple thin-layer analytical model was developed for the change in the chemical composition of the gas in the catalytic region when the catalytic recombination rate coefficient $\gamma_{w}$ takes a realistic value of $O\left(10^{-2}\right)$. Using this model, good predictions of the surface heat flux in the catalytic region can be made using only knowledge of the flow in the non-catalytic case and the catalytic efficiency $\gamma_{w}$. Although we have considered a flat plate, the analytic model should apply to similar situations with an attached boundary layer and an isothermal wall. Further, since the chemistry does not feature at leading order, this model should apply to more complex situations, including those in which there is recombination of more than one species at the surface. Although full numerical solutions of the non-catalytic problems were used in this study, the analytic model could be used to predict the effects of catalysis in any situation where there is sufficient information about the underlying flow. For larger, $O(1)$, values of $\gamma_{w}$ it was shown that the thin-layer model did not apply due to the significant upstream effects arising from the streamwise diffusion terms, which alter the gas composition ahead of the catalytic region. However, the formulae presented should provide useful upper limits on the heat transfer rate for the entire range of $\gamma_{w}$. Note also, that although we considered a change from non-catalytic to catalytic surfaces only, similar behaviour to that found in this study might be expected in any flow with a discontinuous increase in the catalytic efficiency.

The reaction mechanism used was a three-species, three-reaction, dissociation/ recombination scheme. A number of different kinetic schemes taken from the literature were investigated. It was shown that with the basic schemes, the calculated values of the surface heat transfer rate at the catalytic junction did not match the experimental results. However, a modified version of one of the most commonly used kinetic schemes, adjusted to produce more realistic values of the recombination rate at low temperatures, could reproduce the experimental results for the non-catalytic materials within the uncertainties in the data. This modified scheme was used to estimate $\gamma_{w}$ by matching the numerical and experimental heat fluxes. The predicted values have a large amount of scatter. They are, however, consistent with those found in the literature.

One of the major problems in comparing experimental and theoretical results for problems of the type considered in this study is in determining appropriate values of $\gamma_{w}$ and of the forward and backward reaction rates, particularly at low temperatures. 
Even at higher temperatures there is a threefold difference in the reaction rates given by Park (1984) and Kang \& Dunn (1972), similar to the variation we have found in $\gamma_{w}$ (table 9). Most kinetic schemes for reaction mechanisms of the type considered in this study assume higher temperatures $(>1000 \mathrm{~K})$ than the wall temperature used here. For many applications, e.g. re-entry spacecraft, it may not be necessary to consider such low temperatures. However, most experimental high-speed flow facilities used for this type of study have very short run times (less than $10^{-3} \mathrm{~s}$ ), and the standard assumption made when interpreting the results from such facilities is that the surface of the test body is isothermal at the ambient temperature. Clearly there is a need for accurate and reliable estimates of the surface and gas reaction rates if comparisons of the type attempted here are to produce better agreement.

The authors would like to thank Dr P. Batten of UMIST and Professor R. A. East of the University of Southampton for their advice. This work was funded by the United Kingdom Engineering and Physical Science Research Council (EPSRC) through a research studentship award, and provision of computing facilities. They would also like to thank the referees for their helpful suggestions.

\section{REFERENCES}

Amaratunga, S. R. 1998 A numerical study into surface catalytic effects in non-equilibrium reacting viscous hypersonic flow. PhD thesis, University of Southampton.

ANDERSON, L. A. 1973 Effect of surface catalytic activity on stagnation heat-transfer rates. AIAA J. 11, 649-656.

Anderson, J. D. JR 1989 Hypersonic and High Temperature Gas Dynamics. McGraw-Hill.

Balakrishnan, A. 1986 Correlations for specific heats of air species to 50,000 K. AIAA Paper 86-1277.

Batten, P., Clarke, N., Lambert, C \& Causon, D. 1996 On the choice of wavespeeds for HLlC Riemann solvers. SIAM J. Sci. Comput. 18, 1553-1570.

Batten, P., Ingram, D. M., Saunders, R. \& Causon, D. M. 1996 A time-splitting approach to solving the Navier-Stokes equations. Computers Fluids, 25, 421-431.

Chen, Y. K. \& Chandler, G. V. 1993 Navier-Stokes solutions with surface catalysis for Martian atmospheric entry. J. Spacecraft Rockets 30, 32-42.

Chung, P. M. \& Anderson, A. D. 1961 Heat transfer to surfaces of finite catalytic activity in frozen dissociated hypersonic flow. NASA TN D-350.

Chung, P. M. 1965 Chemically reacting non-equilibrium boundary layers. Adv. Heat Transfer 2, 109-270.

Dorrance, W. H. 1962 Viscous Hypersonic Flow. McGraw-Hill.

East, R. A., Stalker, R. J. \& Baird, J. P. 1980 Measurements of heat transfer to a flat plate in a dissociated high enthalpy flow. J. Fluid Mech. 97, 673-699.

ECKERT, E. R. G. 1955 Engineering relations for skin friction and heat transfer to surfaces in high velocity flows. J. Aerospace Sci. 22.

EINFELDT, B. 1988 On Godunov-type methods for gas dynamics. SIAM J. Numer. Anal. 25, 294-318.

FAY, J. A. \& Riddell, F. R. 1958 Theory of stagnation point heat transfer in dissociated air. J. Aeronaut. Sci. 25, 73-85.

Godunov, S. K. 1959 A Difference method for the numerical calculation of discontinuous solutions of hydrodynamic equations. Mat. Sbornik. 47, 271-290.

GOUlARD, R. 1958 On catalytic recombination rates in hypersonic stagnation heat transfer. Jet Propulsion 28, 737-745.

Grumet, A. A., Anderson, J. D. Jr \& Lewis, M. J. 1991 A numerical study of shock wave/boundary layer interaction in non-equilibrium chemically reacting air: the effects of catalytic walls. $A I A A$ Paper 91-0245.

Harten, A., Lax, P. D., Van Leer, B. 1983 On upstream differencing and Godunov-type schemes for hyperbolic conservation laws. SIAM Rev. 25, 35-61. 
High Speed Flow Fields (HSFF) Workshop, University of Houston, Texas, 1995. http:// hhsfd.math.uh.edu/

INGER, G. R. 1963 Non-equilibrium stagnation point boundary layers with arbitrary surface catalysis. AIAA J. 1, 1776-1784

Kang, S. W. \& DunN, M. G. 1972 Theoretical and measured electron-density distributions for the RAM vehicle at high altitudes. AIAA Paper 72-689.

Kee, R. J., Rupley, F. M. \& Miller, J. A. 1987 The Chemkin Thermodynamic Data Base. Sandia National Labs., Livermore, CA.

LEES, L. 1956 Laminar heat transfer over blunt nosed bodies at hypersonic flight. Jet Propulsion 26, 259-269.

Miller, J. H., Tannehill, J. C., Wadawadigi, G., Edwards, T. A. \& Lawrence, S. L. 1995 Computation of hypersonic flow with finite catalytic walls. J. Thermophys. Heat Transfer 9, 486-493.

Murray, P. R. S. 1982 Advanced Chemistry. Pan Books Ltd, London.

PARK, C. 1984 Problems of rate chemistry in the flight regimes of aeroassisted orbital transfer vehicles. AIAA Paper 84-1730.

PARK, C. 1985 On convergence of computation of chemically reacting flows. AIAA Paper 85-0247.

Reid, R. C., Prausnitz, J. M. \& Sherwood, T. K. 1977 The Properties of Gases and Liquids, 3rd Edn. McGraw-Hill.

ScotT, C. D. 1985 Effect of non-equilibrium and wall catalysis on shuttle heat transfer. J. Spacecraft Rockets 22, 489-499.

Stewart, D. A., Rakich, J. V. \& Lanfranco, M. J. 1983 Catalytic surface effects on space shuttle thermal protection system during earth re-entry of flights STS-2 through STS-5. NASA CP 2283.

Stull, D. R. \& Prophet, H. 1971 JANAF Thermochemical Tables, second edn. NSRDS-NBS 37, National Bureau of Standards, Washington DC.

Toro, E. F., Spruce, M. \& SpEARES, W. 1994 Restoration of the contact surface in the HLL-Riemann solver. Shock Waves 4, 25-34.

VAN LEER, B. 1979 Towards the ultimate conservative difference scheme. V. A second-order sequel to Godunov. J. Comput. Phys. 32, 101-136.

Vincenti, W. G. \& Kruger, C. H. JR 1965 Introduction to Physical Gas Dynamics. Krieger, Malabar. Wilke, C. R. 1950 A viscosity equation for gas mixtures. J. Chem. Phys. 18. 